\title{
Monodromy Problem and the Boundary Condition for Some Painlevé Equations
}

By

\author{
Michio JIMBO*
}

\section{Introduction}

The Painlevé transcendents and their generalizations have aroused much interest among mathematicians and mathematical physicists in recent years ([1]-[8]). Yet their properties as special functions still remain almost unexplored. For example, the problem of determining their behavior at the fixed critical singularities is left open, except for a few special cases ([3][4]). In the present paper we treat this problem for the following three kinds of Painlevé equations at the fixed critical point $t=0$ :

$$
\begin{aligned}
\frac{d^{2} y}{d t^{2}}=\frac{1}{y}\left(\frac{d y}{d t}\right)^{2}-\frac{1}{t} \frac{d y}{d t}+\frac{1}{t}\left(\alpha y^{2}+\beta\right) & +\gamma y^{3}+\frac{\delta}{y} \\
\frac{d^{2} y}{d t^{2}}=\left(\frac{1}{2 y}+\frac{1}{y-1}\right)\left(\frac{d y}{d t}\right)^{2}-\frac{1}{t} \frac{d y}{d t}+ & \frac{(y-1)^{2}}{t^{2}}\left(\alpha y+\frac{\beta}{y}\right) \\
& +\frac{\gamma y}{t}+\frac{\delta y(y+1)}{y-1}
\end{aligned}
$$

$$
\begin{aligned}
\frac{d^{2} y}{d t^{2}}= & \frac{1}{2}\left(\frac{1}{y}+\frac{1}{y-1}+\frac{1}{y-t}\right)\left(\frac{d y}{d t}\right)^{2}-\left(\frac{1}{t}+\frac{1}{t-1}+\frac{1}{y-t}\right) \frac{d y}{d t} \\
& +\frac{y(y-1)(y-t)}{t^{2}(t-1)^{2}}\left(\alpha+\beta \frac{t}{y^{2}}+\gamma \frac{t-1}{(y-1)^{2}}+\delta \frac{t(t-1)}{(y-t)^{2}}\right) .
\end{aligned}
$$

As is well known ([9][7]), the Painlevé equations arise as monodromy preserving deformation equations for a $2 \times 2$ system of linear differential equations

$$
\frac{d Y}{d x}=A(x, t) Y, \quad A(x, t): \text { rational in } x .
$$

A particular Painlevé transcendent is specified by the monodromy data for $(0.1)$.

Received November 26, 1981.

* Research Institute for Mathematical Sciences, Kyoto University, Kyoto 606, Japan. 
Now let $\tau(t)$ denote the associated $\tau$ function ([10][7]), say, for (PVI). At $t=0$ it is shown to have the asymptotic expansion of the form

$$
\begin{aligned}
\tau(t) & \sim \text { const. } t^{\left(\sigma^{2}-\theta_{0}^{2}-\theta_{t}^{2}\right) / 4} \\
\times & \left(1+\frac{1}{8 \sigma^{2}}\left(\theta_{o}^{2}-\theta_{t}^{2}-\sigma^{2}\right)\left(\theta_{\infty}^{2}-\theta_{1}^{2}-\sigma^{2}\right) t\right. \\
& -\frac{\hat{s}}{16 \sigma^{2}(1+\sigma)^{2}}\left(\theta_{o}^{2}-\left(\theta_{t}-\sigma\right)^{2}\right)\left(\theta_{\infty}^{2}-\left(\theta_{1}-\sigma\right)^{2}\right) t^{1+\sigma} \\
& -\frac{\hat{s}^{-1}}{16 \sigma^{2}(1-\sigma)^{2}}\left(\theta_{o}^{2}-\left(\theta_{t}+\sigma\right)^{2}\right)\left(\theta_{\infty}^{2}-\left(\theta_{1}+\sigma\right)^{2}\right) t^{1-\sigma} \\
& \left.+\sum_{j=2}^{\infty} \sum_{|k| \leqq j} a_{j k} t^{j-k \sigma}\right)
\end{aligned}
$$

where $\theta_{v}(v=0,1, t, \infty)$ are related to the coefficients $\alpha, \beta, \gamma, \delta$ in (PVI), and $\sigma, \hat{s}$ are the two integration constants. The main result of this paper is an explicit formula which gives these integration constants in terms of the monodromy data for (0.1). Similar formulas are derived also for (PIII) and (PV). In the case of (PVI), the fixed critical points $t=0,1$ and $\infty$ play equivalent rôles. Hence the result above makes it possible to derive a connection formula for the $\tau$ function for (PVI).

This paper is planned as follows. In Section 1 we state the result for (PVI). Its derivation is given in Section 2. The method employed here is to study the linear differential equation (0.1) in the limit $t \rightarrow 0$. We show that the determination of the asymptotic expansion is reduced to a connection problem for the limiting differential equations, which can be solved in the case of (PVI) in terms of hypergeometric functions.

In Section 3 we give the results for (PIII) and (PV). As an application we derive the short distance expansion of the $\tau$ function which appears in the 2 point correlation function of the Federbush model ([5] IV, Supplement to IV). In a special case, this reproduces the result of McCoy-Tracy-Wu [3] (for $v=0$ ).

\section{Acknowledgement}

The author would like to thank Professor T. Miwa and Professor K. Takano for helpful conversations and encouragement. 


\section{§1. The Sixth Painlevé Equation}

Following [7] II, we first recall the setting of the monodromy problem for PVI. We consider a $2 \times 2$ system of linear differential equations

$$
\begin{aligned}
& \frac{\partial Y}{\partial x}=\left(\frac{A_{0}(t)}{x}+\frac{A_{1}(t)}{x-1}+\frac{A_{t}(t)}{x-t}\right) Y \equiv A(x, t) Y \\
& \frac{\partial Y}{\partial t}=-\frac{A_{t}(t)}{x-t} Y .
\end{aligned}
$$

Without loss of generality we may assume that

$$
\begin{aligned}
& A_{0}(t)+A_{1}(t)+A_{t}(t)=-\frac{1}{2}\left(\begin{array}{ll}
\theta_{\infty} & \\
& -\theta_{\infty}
\end{array}\right) \\
& \text { the eigenvalues of } A_{v}(t)= \pm \frac{1}{2} \theta_{v} \quad(v=0,1, t),
\end{aligned}
$$

where $\theta_{v}$ are constants. If we write the $(1,2)$-th component of $A(x, t)$ as $(A(x, t))_{12}=k(t)(x-y(t)) / x(x-1)(x-t)$, then $y=y(t)$ satisfies the sixth Painlevé equation (PVI) with the parameters

$$
\alpha=\frac{1}{2}\left(\theta_{\infty}-1\right)^{2}, \quad \beta=-\frac{1}{2} \theta_{0}^{2}, \quad \gamma=\frac{1}{2} \theta_{1}^{2}, \quad \delta=\frac{1}{2}\left(1-\theta_{t}^{2}\right) .
$$

By definition, the $\tau$ function $\tau(t)$ for PVI is given by

$$
\frac{d}{d t} \log \tau(t)=\operatorname{tr}\left(\frac{A_{0}(t)}{t}+\frac{A_{1}(t)}{t-1}\right) A_{t}(t) .
$$

Setting

$$
\zeta(t)=t(t-1) \frac{d}{d t} \log \tau(t)+\frac{t}{4}\left(\theta_{t}^{2}-\theta_{\infty}^{2}\right)-\frac{1}{8}\left(\theta_{t}^{2}+\theta_{0}^{2}-\theta_{\infty}^{2}-\theta_{1}^{2}\right)
$$

we have the differential equation

$$
\begin{aligned}
& \frac{d \zeta}{d t}\left(t(t-1) \frac{d^{2} \zeta}{d t^{2}}\right)^{2}+\left[2 \frac{d \zeta}{d t}\left(t \frac{d \zeta}{d t}-\zeta\right)-\left(\frac{d \zeta}{d t}\right)^{2}\right. \\
& \left.\quad-\frac{1}{16}\left(\theta_{t}^{2}-\theta_{\infty}^{2}\right)\left(\theta_{0}^{2}-\theta_{1}^{2}\right)\right]^{2} \\
& =\left(\frac{d \zeta}{d t}+\frac{1}{4}\left(\theta_{t}+\theta_{\infty}\right)^{2}\right)\left(\frac{d \zeta}{d t}+\frac{1}{4}\left(\theta_{t}-\theta_{\infty}\right)^{2}\right)\left(\frac{d \zeta}{d t}+\frac{1}{4}\left(\theta_{0}+\theta_{1}\right)^{2}\right) \\
& \quad \times\left(\frac{d \zeta}{d t}+\frac{1}{4}\left(\theta_{0}-\theta_{1}\right)^{2}\right)
\end{aligned}
$$


In the sequel we assume

$(\mathrm{A} 1)_{\mathrm{PVI}}$

$$
\theta_{0}, \theta_{1}, \theta_{t}, \theta_{\infty} \notin \mathbb{Z}
$$

Let $Y=Y(x, t)$ be the fundamental solution matrix of (1.1) normalized at $x=\infty$. Its local behavior at the singularities reads as follows.

$$
\begin{aligned}
Y(x, t) & =\left(G^{(0)}(t)+O(x)\right) x^{\frac{1}{2}\left(\theta_{0}-\theta_{0}\right)} C^{(0)} & & (x \rightarrow 0) \\
& =\left(G^{(1)}(t)+O(x-1)\right)(x-1)^{\frac{1}{2}\left(\theta^{\theta_{1}}-\theta_{1}\right)} C^{(1)} & & (x \rightarrow 1) \\
& =\left(G^{(t)}(t)+O(x-t)\right)(x-t)^{\frac{1}{2}\left(\theta_{t}-\theta_{t}\right)} C^{(t)} & & (x \rightarrow t) \\
& =\left(1+O\left(x^{-1}\right)\right) x^{-\frac{1}{2}\left(\theta_{\infty}-\theta_{\infty}\right)} & & (x \rightarrow \infty) .
\end{aligned}
$$

Here $G^{(v)}(t), C^{(v)}$ are invertible matrices. In particular, the connection matrices $C^{(v)}$ are independent of $t$. The monodromy matrices $M^{(v)}=$ $C^{(v)-1}\left(\begin{array}{c}e^{\pi i \theta_{v}} \\ e^{-\pi i \theta_{v}}\end{array}\right) C^{(v)} \in S L(2, C)$ satisfy the relation

$$
M^{(\infty)} M^{(1)} M^{(t)} M^{(0)}=1 .
$$

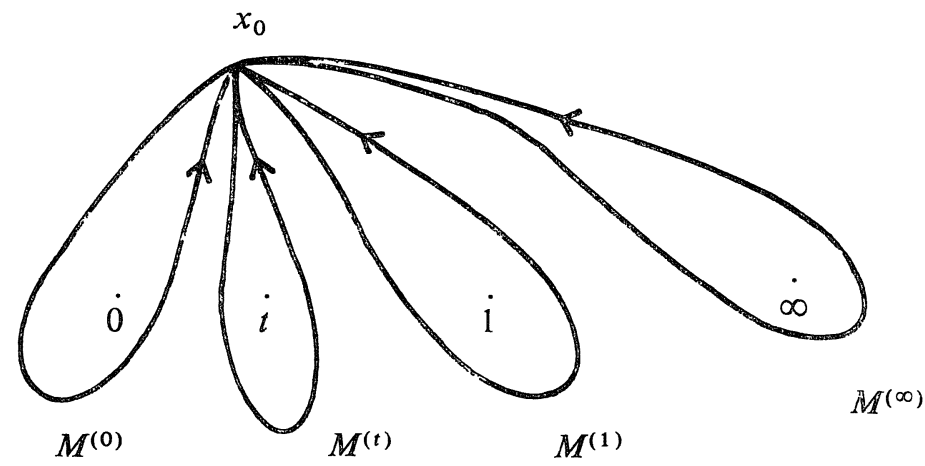

Figure. The choice of paths corresponding to $M^{(\nu)}$.

Put $p_{v}=\operatorname{tr} M^{(v)}=2 \cos \pi \theta_{v}, p_{\mu \nu}=\operatorname{tr} M^{(\mu)} M^{(v)}=p_{v \mu}$. We have then the following one relation among them:

$$
\begin{aligned}
0= & p_{01} p_{1 t} p_{t 0}+p_{01}^{2}+p_{1 t}^{2}+p_{t 0}^{2} \\
& -\left(p_{0} p_{1}+p_{t} p_{\infty}\right) p_{01}-\left(p_{1} p_{t}+p_{0} p_{\infty}\right) p_{1 t}-\left(p_{t} p_{0}+p_{1} p_{\infty}\right) p_{t 0} \\
& +p_{0}^{2}+p_{1}^{2}+p_{t}^{2}+p_{\infty}^{2}+p_{0} p_{1} p_{t} p_{\infty}-4
\end{aligned}
$$

It is sometimes convenient to write $p_{\mu \nu}=2 \cos \pi \sigma_{\mu \nu}$ with $\sigma_{\mu \nu}=\sigma_{v \mu} \in \mathbf{C}, 0 \leqq$ $\operatorname{Re} \sigma_{\mu \nu} \leqq 1$.

Our aim is to determine the asymptotic behavior of $\tau(t)$ as $t \rightarrow 0$. Here we assume further that 
(A.2) $)_{\mathrm{PVI}} \quad 0 \leqq \operatorname{Re} \sigma_{t o}<1$,

(A.3) $)_{\mathrm{PVI}} \quad \frac{1}{2}\left(\theta_{0} \pm \theta_{t} \pm^{\prime} \sigma_{t 0}\right), \frac{1}{2}\left(\theta_{\infty} \pm \theta_{1} \pm^{\prime} \sigma_{t 0}\right) \notin \mathbb{Z}$.

Under these conditions, we can parametrize the monodromy matrices satisfying (1.5) as follows. When $\sigma=\sigma_{t 0} \neq 0$, we have

$$
\begin{aligned}
& M^{(1)} \\
& =\frac{1}{i \sin \pi \theta_{\infty}}\left(\begin{array}{l}
\cos \pi \sigma-e^{-\pi i \theta_{\infty}} \cos \pi \theta_{1} \\
2 r^{-1} e^{\pi i \theta_{\infty}} \sin \frac{\pi}{2}\left(\theta_{\infty}-\theta_{1}+\sigma\right) \sin \frac{\pi}{2}\left(\theta_{\infty}-\theta_{1}-\sigma\right) \\
-2 r e^{-\pi i \theta_{\infty}} \sin \frac{\pi}{2}\left(\theta_{\infty}+\theta_{1}+\sigma\right) \sin \frac{\pi}{2}\left(\theta_{\infty}+\theta_{1}-\sigma\right) \\
-\cos \pi \sigma+e^{\pi i \theta_{\infty}} \cos \pi \theta_{1}
\end{array}\right)
\end{aligned}
$$

$C M^{(t)} C^{-1}$

$$
\begin{aligned}
& =\frac{1}{i \sin \pi \sigma}\left(\begin{array}{l}
e^{\pi i \sigma} \cos \pi \theta_{t}-\cos \pi \theta_{0} \\
2 s^{-1} e^{-\pi i \sigma} \sin \frac{\pi}{2}\left(\theta_{0}+\theta_{t}+\sigma\right) \sin \frac{\pi}{2}\left(\theta_{0}-\theta_{t}-\sigma\right)
\end{array}\right. \\
& \begin{array}{l}
-2 s e^{\pi i \sigma} \sin \frac{\pi}{2}\left(\theta_{0}+\theta_{t}-\sigma\right) \sin \frac{\pi}{2}\left(\theta_{0}-\theta_{t}+\sigma\right) \\
-e^{-\pi i \sigma} \cos \pi \theta_{t}+\cos \pi \theta_{0}
\end{array}
\end{aligned}
$$

$C M^{(0)} C^{-1}$

$$
\begin{aligned}
& =\frac{1}{i \sin \pi \sigma}\left(\begin{array}{l}
e^{\pi i \sigma} \cos \pi \theta_{0}-\cos \pi \theta_{t} \\
-2 s^{-1} \sin \frac{\pi}{2}\left(\theta_{0}-\theta_{t}-\sigma\right) \sin \frac{\pi}{2}\left(\theta_{0}+\theta_{t}+\sigma\right)
\end{array}\right. \\
& \left.\begin{array}{l}
2 s \sin \frac{\pi}{2}\left(\theta_{0}+\theta_{t}-\sigma\right) \sin \frac{\pi}{2}\left(\theta_{0}-\theta_{t}+\sigma\right) \\
-e^{-\pi i \sigma} \cos \pi \theta_{0}+\cos \pi \theta_{t}
\end{array}\right)
\end{aligned}
$$

where

$$
C=\left(\begin{array}{cc}
\sin \frac{\pi}{2}\left(\theta_{\infty}-\theta_{1}-\sigma\right) & r \sin \frac{\pi}{2}\left(\theta_{\infty}+\theta_{1}+\sigma\right) \\
r^{-1} \sin \frac{\pi}{2}\left(\theta_{\infty}-\theta_{1}+\sigma\right) & \sin \frac{\pi}{2}\left(\theta_{\infty}+\theta_{1}-\sigma\right)
\end{array}\right)
$$

and $r, s \neq 0$. In terms of the invariants $2 \cos \pi \sigma_{\mu \nu}=\operatorname{tr} M^{(\mu)} M^{(v)}$, the definition of the parameter $s$ reads

$$
\begin{aligned}
4 s^{ \pm 1} \sin \frac{\pi}{2}\left(\theta_{0}+\theta_{t} \mp \sigma\right) & \sin \frac{\pi}{2}\left(\theta_{0}-\theta_{t} \pm \sigma\right) \\
& \times \sin \frac{\pi}{2}\left(\theta_{\infty}+\theta_{1} \mp \sigma\right) \sin \frac{\pi}{2}\left(\theta_{\infty}-\theta_{1} \mp \sigma\right)
\end{aligned}
$$




$$
\begin{aligned}
= & \left( \pm i \sin \pi \sigma \cos \pi \sigma_{1 t}-\cos \pi \theta_{t} \cos \pi \theta_{\infty}-\cos \pi \theta_{0} \cos \pi \theta_{1}\right) e^{ \pm \pi i \sigma} \\
& \pm i \sin \pi \sigma \cos \pi \sigma_{01}+\cos \pi \theta_{t} \cos \pi \theta_{1}+\cos \pi \theta_{\infty} \cos \pi \theta_{0} .
\end{aligned}
$$

When $\sigma=\sigma_{t 0}=0$, the parametrization for $M^{(t)}, M^{(0)}$ is obtained by setting $s=1+s_{1} \sigma$ and letting $\sigma \rightarrow 0$. Namely we introduce $s_{1} \in \mathbb{C}$ by

$$
\begin{aligned}
(1.7)^{\prime} \quad M^{(t)}= & C^{(t)-1}\left(\begin{array}{cc}
e^{\pi i \theta_{t}} & 0 \\
0 & e^{-\pi i \theta_{t}}
\end{array}\right) C^{(t)}, M^{(0)}=C^{(0)-1}\left(\begin{array}{cc}
e^{\pi i \theta_{0}} & 0 \\
0 & e^{-\pi i \theta_{0}}
\end{array}\right) C^{(0)}, \\
\left(C^{(t)}\right)_{11}= & -\left(s_{1} \sin \frac{\pi}{2}\left(\theta_{\infty}-\theta_{1}\right)+\pi e^{\frac{\pi i}{2}\left(\theta_{\infty}-\theta_{1}\right)}\right) \\
& \times \sin \frac{\pi}{2}\left(\theta_{0}+\theta_{t}\right) \sin \frac{\pi}{2}\left(-\theta_{0}+\theta_{t}\right)+\pi \sin \pi \theta_{t} \sin \frac{\pi}{2}\left(\theta_{\infty}-\theta_{1}\right) \\
\left(C^{(t)}\right)_{12}= & r\left[-\left(s_{1} \sin \frac{\pi}{2}\left(\theta_{\infty}+\theta_{1}\right)-\pi e^{-\frac{\pi i}{2}\left(\theta_{\infty}+\theta_{1}\right)}\right)\right. \\
& \left.\times \sin \frac{\pi}{2}\left(\theta_{0}+\theta_{t}\right) \sin \frac{\pi}{2}\left(-\theta_{0}+\theta_{t}\right)+\pi \sin \pi \theta_{t} \sin \frac{\pi}{2}\left(\theta_{\infty}+\theta_{1}\right)\right] \\
\left(C^{(t)}\right)_{21}= & r^{-1}\left(s_{1} \sin \frac{\pi}{2}\left(\theta_{\infty}-\theta_{1}\right)+\pi e^{\frac{\pi i}{2}\left(\theta_{\infty}-\theta_{1}\right)}\right) \\
\left(C^{(t)}\right)_{22}= & s_{1} \sin \frac{\pi}{2}\left(\theta_{\infty}+\theta_{1}\right)-\pi e^{-\frac{\pi i}{2}\left(\theta_{\infty}+\theta_{1}\right)}, \\
\left(C^{(0)}\right)_{11}= & -s_{1} \sin \frac{\pi}{2}\left(\theta_{0}+\theta_{t}\right) \sin \frac{\pi}{2}\left(\theta_{\infty}-\theta_{1}\right)-\pi \sin \frac{\pi}{2}\left(\theta_{0}+\theta_{t}-\theta_{\infty}+\theta_{1}\right) \\
\left(C^{(0)}\right)_{12}= & r\left(-s_{1} \sin \frac{\pi}{2}\left(\theta_{0}+\theta_{t}\right) \sin \frac{\pi}{2}\left(\theta_{\infty}+\theta_{1}\right)+\pi \sin \frac{\pi}{2}\left(\theta_{0}+\theta_{t}+\theta_{\infty}+\theta_{1}\right)\right) \\
\left(C^{(0)}\right)_{21}= & r^{-1}\left(s_{1} \sin \frac{\pi}{2}\left(-\theta_{0}+\theta_{t}\right) \sin \frac{\pi}{2}\left(\theta_{\infty}-\theta_{1}\right)\right. \\
& \left.\quad+\pi \sin \frac{\pi}{2}\left(-\theta_{0}+\theta_{t}-\theta_{\infty}+\theta_{1}\right)\right) \\
\left(C^{(0)}\right)_{22}= & s_{1} \sin \frac{\pi}{2}\left(-\theta_{0}+\theta_{t}\right) \sin \frac{\pi}{2}\left(\theta_{\infty}+\theta_{1}\right) \\
& -\pi \frac{\pi}{2}\left(-\theta_{0}+\theta_{t}+\theta_{\infty}+\theta_{1}\right) .
\end{aligned}
$$

Now we can state the main result for PVI.

Theorem 1.1. Under the assumptions (A.1 $)_{\mathrm{PVI}}-(\mathrm{A} .3)_{\mathrm{PVI}}$, we have the following asymptotic expansion of the $\tau$ function as $t \rightarrow 0$.

$$
\begin{aligned}
& \tau(t) \sim \text { const. } t^{\left(\sigma^{2}-\theta_{0}^{2}-\theta_{t}^{2}\right) / 4} \\
& \times\left[1+\frac{1}{8 \sigma^{2}}\left(\theta_{0}^{2}-\theta_{t}^{2}-\sigma^{2}\right)\left(\theta_{\infty}^{2}-\theta_{1}^{2}-\sigma^{2}\right) t\right. \\
& -\frac{\hat{s}}{16 \sigma^{2}(1+\sigma)^{2}}\left(\theta_{0}^{2}-\left(\theta_{t}-\sigma\right)^{2}\right)\left(\theta_{\infty}^{2}-\left(\theta_{1}-\sigma\right)^{2}\right) t^{1+\sigma}
\end{aligned}
$$




$$
\begin{aligned}
& -\frac{\hat{s}^{-1}}{16 \sigma^{2}(1-\sigma)^{2}}\left(\theta_{0}^{2}-\left(\theta_{t}+\sigma\right)^{2}\right)\left(\theta_{\infty}^{2}-\left(\theta_{1}+\sigma\right)^{2}\right) t^{1-\sigma} \\
& \left.+O\left(|t|^{2(1-\mathrm{Re} \sigma)}\right)\right]
\end{aligned}
$$

where $\sigma \neq 0$ and $\hat{s}$ is related to $s$ in (1.8) through

$$
\begin{gathered}
\hat{s}=\frac{\Gamma(1-\sigma)^{2} \Gamma\left(\frac{1}{2}\left(\theta_{0}+\theta_{t}+\sigma\right)+1\right) \Gamma\left(\frac{1}{2}\left(-\theta_{0}+\theta_{t}+\sigma\right)+1\right)}{\Gamma(1+\sigma)^{2} \Gamma\left(\frac{1}{2}\left(\theta_{0}+\theta_{t}-\sigma\right)+1\right) \Gamma\left(\frac{1}{2}\left(-\theta_{0}+\theta_{t}-\sigma\right)+1\right)} \\
\times \frac{\Gamma\left(\frac{1}{2}\left(\theta_{\infty}+\theta_{1}+\sigma\right)+1\right) \Gamma\left(\frac{1}{2}\left(-\theta_{\infty}+\theta_{1}+\sigma\right)+1\right)}{\Gamma\left(\frac{1}{2}\left(\theta_{\infty}+\theta_{1}-\sigma\right)+1\right) \Gamma\left(\frac{1}{2}\left(-\theta_{\infty}+\theta_{1}-\sigma\right)+1\right)} s .
\end{gathered}
$$

If $\sigma=0$, then

$$
\begin{aligned}
& \tau(t) \sim \text { const. } t^{-\left(\theta_{0}^{2}+\theta_{t}^{2}\right) / 4} \\
& \times\left[1-\frac{1}{16}\left(\theta_{\infty}^{2}-\theta_{1}^{2}\right)\left(\theta_{0}^{2}-\theta_{t}^{2}\right) t\left(\Omega^{2}+2 \Omega+3\right)\right. \\
& +\frac{1}{4}\left(\theta_{t}\left(\theta_{\infty}^{2}-\theta_{1}^{2}\right)+\theta_{1}\left(\theta_{0}^{2}-\theta_{t}^{2}\right)\right) t(\Omega+1) \\
& -\frac{1}{2} \theta_{1} \theta_{t} t \\
& +o(|t|)]
\end{aligned}
$$

with $\Omega=1-\hat{s}_{1}-\log t$, and $\hat{s}_{1}$ is given by

$$
\begin{aligned}
\hat{s}_{1} & =s_{1}+\psi\left(\frac{1}{2}\left(\theta_{0}+\theta_{t}\right)+1\right)+\psi\left(\frac{1}{2}\left(-\theta_{0}+\theta_{t}\right)+1\right) \\
& +\psi\left(\frac{1}{2}\left(\theta_{\infty}+\theta_{1}\right)+1\right)+\psi\left(\frac{1}{2}\left(-\theta_{\infty}+\theta_{1}\right)+1\right)-4 \psi(1) .
\end{aligned}
$$

Here $\psi(x)=-\frac{d}{d x} \log \Gamma(x)$ denotes the diGamma function.

More precisely, for any $\varphi>0$, there exists an $\varepsilon>0$ such that (1.7)-(1.7)' holds as $t \rightarrow 0$ in the sector $\{t \in \mathbb{C}|0<| t|<\varepsilon,| \arg t \mid<\varphi\}$.

Higher order expansion is determined from the equation (1.3).

The $\tau$ function is uniquely specified by the exponents $\theta_{v}$ and the monodromy matrices $M^{(v)}$. In order to signify the dependence on them, we employ the notation $\tau\left(t ; \theta_{0}, \theta_{t}, \theta_{1}, \theta_{\infty} ; M^{(0)}, M^{(t)}, M^{(1)}\right)$. Actually it is invariant under the joint similarity transformation $M^{(v)} \mapsto P M^{(v)} P^{-1}(v=0, t, 1)$ so that the 
$M^{(v)}$ dependence enters only through the invariants $\operatorname{tr} M^{(\mu)} M^{(v)}$.

Since the regular singularities $x=0,1, t, \infty$ play equivalent rôles, one can exchange them by a fractional linear transformation. Correspondingly one has the transformation law for the $\tau$ function

$$
\begin{aligned}
& \tau\left(1-t ; \theta_{0}, \theta_{t}, \theta_{1}, \theta_{\infty} ; M^{(0)}, M^{(t)}, M^{(1)}\right) \\
&=\text { const. } \tau\left(t ; \theta_{1}, \theta_{t}, \theta_{0}, \theta_{\propto} ; M^{(1)}, M^{(t)}, M^{(0)}\right), \\
& \tau\left(t^{-1} ; \theta_{0}, \theta_{t}, \theta_{1}, \theta_{\infty} ; M^{(0)}, M^{(t)}, M^{(1)}\right) \\
&=\text { const. } t_{t}^{\theta_{t}^{2} / 2} \tau\left(t ; \theta_{\infty}, \theta_{t}, \theta_{1}, \theta_{0} ; \tilde{M}^{(0)}, \tilde{M}^{(t)}, \tilde{M}^{(1)}\right)
\end{aligned}
$$

where $\tilde{M}^{(0)}=M^{(\infty)}, \tilde{M}^{(t)}=\left(M^{(\infty)} M^{(1)}\right) M^{(t)}\left(M^{(\infty)} M^{(1)}\right)^{-1}$ and $\tilde{M}^{(1)}=\left(M^{(\infty)} M^{(1)}\right.$ $\left.\times M^{(t)}\right) M^{(1)}\left(M^{(\infty)} M^{(1)} M^{(t)}\right)^{-1}$, up to a common similarity factor (note the convention for the choice of paths shown in Fig.). Combining this with Theorem 1.1, one has thus the following connection formula.

Theorem 1.2. Suppose $\tau(t)=\tau\left(t ; \theta_{0}, \theta_{t}, \theta_{1}, \theta_{\infty} ; M^{(0)}, M^{(t)}, M^{(1)}\right)$ has the behavior (1.9) at $t=0$ with some $s$ and $\sigma=\sigma_{0 t}$. Let $\sigma_{01}$ and $\sigma_{1 t}$ be defined through (1.6), (1.8). We assume

$$
\begin{aligned}
0 \leqq & \operatorname{Re} \sigma_{\mu \nu}<1, \sigma_{\mu \nu} \neq 0 \quad(\mu \neq v, \mu, v=0,1, t) \\
& \frac{1}{2}\left(\theta_{0} \pm \theta_{t} \pm^{\prime} \sigma_{0 t}\right), \frac{1}{2}\left(\theta_{\infty} \pm \theta_{1} \pm^{\prime} \sigma_{o t}\right), \frac{1}{2}\left(\theta_{1} \pm \theta_{t} \pm^{\prime} \sigma_{1 t}\right), \\
& \frac{1}{2}\left(\theta_{\infty} \pm \theta_{0} \pm^{\prime} \sigma_{1 t}\right), \frac{1}{2}\left(\theta_{\infty} \pm \theta_{t} \pm^{\prime} \sigma_{01}\right), \frac{1}{2}\left(\theta_{0} \pm \theta_{1} \pm^{\prime} \sigma_{01}\right) \notin \mathbb{Z} .
\end{aligned}
$$

Then the following asymptotic expansions are valid at $t=1, \infty$ :

$$
\begin{aligned}
& \tau(t) \sim \text { const. }(1-t)^{\left(\sigma_{1 t}^{2}-\theta_{1}^{2}-\theta_{t}^{2}\right) / 4} \\
& \times\left[1+\frac{1}{8 \sigma_{1 t}^{2}}\left(\theta_{1}^{2}-\theta_{t}^{2}-\sigma_{1 t}^{2}\right)\left(\theta_{\infty}^{2}-\theta_{0}^{2}-\sigma_{1 t}^{2}\right)(1-t)\right. \\
& -\frac{\hat{s}_{1 t}}{16 \sigma_{1 t}^{2}\left(1+\sigma_{1 t}\right)^{2}}\left(\theta_{1}^{2}-\left(\theta_{t}-\sigma_{1 t}\right)^{2}\right)\left(\theta_{\infty}^{2}-\left(\theta_{0}-\sigma_{1 t}\right)^{2}\right)(1-t)^{1+\sigma_{1 t}} \\
& -\frac{\hat{s}_{1 t}^{-1}}{16 \sigma_{1 t}^{2}\left(1-\sigma_{1 t}\right)^{2}}\left(\theta_{1}^{2}-\left(\theta_{t}+\sigma_{1 t}\right)^{2}\right)\left(\theta_{\infty}^{2}-\left(\theta_{0}+\sigma_{1 t}\right)^{2}\right)(1-t)^{1-\sigma_{1 t}} \\
& \left.+O\left(|1-t|^{2\left(1-\operatorname{Re} \sigma_{1 t}\right)}\right)\right] \quad(t \rightarrow 1), \\
& \tau(t) \sim \text { const. } t^{-\left(\sigma_{01}^{2}-\theta_{\infty}^{2}+\theta_{t}^{2}\right) / 4} \\
& \times\left[1+\frac{1}{8 \sigma_{01}^{2}}\left(\theta_{\infty}^{2}-\theta_{t}^{2}-\sigma_{01}^{2}\right)\left(\theta_{0}^{2}-\theta_{1}^{2}-\sigma_{01}^{2}\right) t^{-1}\right. \\
& -\frac{\hat{s}_{t \infty}}{16 \sigma_{01}^{2}\left(1+\sigma_{01}\right)^{2}}\left(\theta_{\infty}^{2}-\left(\theta_{t}-\sigma_{01}\right)^{2}\right)\left(\theta_{0}^{2}-\left(\theta_{1}-\sigma_{01}\right)^{2}\right) t^{-1-\sigma_{01}}
\end{aligned}
$$




$$
\begin{aligned}
& -\frac{\hat{s}_{t \infty}^{-1}}{16 \sigma_{01}^{2}\left(1-\sigma_{01}\right)^{2}}\left(\theta_{\infty}^{2}-\left(\theta_{t}+\sigma_{01}\right)^{2}\right)\left(\theta_{0}^{2}-\left(\theta_{1}+\sigma_{01}\right)^{2}\right) t^{-1+\sigma_{01}} \\
& \left.+O\left(|t|^{-2\left(1-\operatorname{Re} \sigma_{01}\right)}\right)\right] \quad(t \rightarrow \infty) .
\end{aligned}
$$

Here $\hat{s}_{1 t}, \hat{s}_{t \infty}$ are obtained by making the following substitutions in (1.8) and (1.10), respectively:

$$
\begin{aligned}
& \hat{s} \longrightarrow \hat{s}_{1 t}, s \longrightarrow s_{1 t}, \theta_{0} \longleftrightarrow \theta_{1}, \sigma \longrightarrow \sigma_{1 t}, \sigma_{1 \imath} \longrightarrow \sigma_{0 t}, \\
& \hat{s} \longrightarrow \hat{s}_{t, \infty}, s \longrightarrow s_{t \infty}, \theta_{\infty} \longleftrightarrow \theta_{0}, \sigma \longrightarrow \sigma_{01}, \sigma_{01} \longrightarrow \tilde{\sigma}_{01}
\end{aligned}
$$

with $\cos \pi \tilde{\sigma}_{01}=\operatorname{tr} \tilde{M}^{(0)} \tilde{M}^{(1)}=-\cos \pi \sigma_{0 t}-2 \cos \pi \sigma_{01} \cos \pi \sigma_{1 t}+2\left(\cos \pi \theta_{0} \cos \pi \theta_{t}\right.$ $\left.+\cos \pi \theta_{\infty} \cos \pi \theta_{1}\right)$.

\section{§2. Derivation}

In this section we show how to derive the formulas (1.9)-(1.9)'.

Let us consider a more general situation

$$
\begin{aligned}
& \frac{\partial Y}{\partial x}=\left(\sum_{\mu=1}^{n_{1}} \frac{A_{\mu}(t)}{x-a_{\mu}}+\sum_{v=1}^{n_{2}} \frac{B_{v}(t)}{x-t b_{v}}\right) Y \\
& \frac{\partial Y}{\partial t}=-\sum_{v=1}^{n_{2}} \frac{b_{v} B_{v}(t)}{x-t b_{v}} Y
\end{aligned}
$$

where $Y, A_{\mu}, B_{v}$ are $m \times m$ matrices, and $a_{\mu}, b_{v}$ are distinct nonzero constants. The integrability of (2.1) leads to the restricted Schlesinger equation

$$
\begin{aligned}
& \frac{d A_{\mu}}{d t}(t)=\sum_{v=1}^{n_{2}} \frac{b_{v}}{t b_{v}-a_{\mu}}\left[B_{v}(t), A_{\mu}(t)\right] \\
& \frac{d B_{v}}{d t}(t)=\frac{1}{t} \sum_{v^{\prime}(\neq v)}\left[B_{v^{\prime}}(t), B_{v}(t)\right]+\sum_{\mu=1}^{n_{1}} \frac{b_{v}}{t b_{v}-a_{\mu}}\left[A_{\mu}(t), B_{v}(t)\right] .
\end{aligned}
$$

We assume $\sum_{\mu=1}^{n_{1}} A_{\mu}(t)+\sum_{v=1}^{n_{2}} B_{v}(t)=-T_{0}^{(\infty)}=$ diagonal, constant, and that each of $A_{\mu}(t), B_{v}(t)$ and $T_{0}^{(\infty)}$ has eigenvalues distinct modulo integers. Let $A_{\mu}^{0}, \widetilde{B}_{v}^{0}$ be constant matrices such that

$$
\sum_{\mu=1}^{n_{1}} A_{\mu}^{0}+\sum_{v=1}^{n_{2}} \widetilde{B}_{v}^{0}=-T_{0}^{(\infty)}
$$

and that the eigenvalues of $A_{\mu}^{0}\left(\operatorname{resp} . \widetilde{B}_{v}^{0}\right)$ coincide with those of $A_{\mu}(t)\left(\operatorname{resp} . B_{v}(t)\right)$. We set $A=\sum_{v=1}^{n_{2}} \widetilde{B}_{v}^{0}$, and denote its eigenvalues by $\mu_{1}, \ldots, \mu_{m}$. The following is known:

Theorem ([5]II). We assume 


$$
\left|\operatorname{Re}\left(\mu_{j}-\mu_{k}\right)\right|<1, j, k=1, \ldots, m .
$$

(i) Choose $\sigma_{1}, K>0$ satisfying $\max _{1 \leqq j, k \leqq m}\left|\operatorname{Re}\left(\mu_{j}-\mu_{k}\right)\right|<\sigma_{1}<1,\left|A_{\mu}^{0}\right|,\left|\widetilde{B}_{v}^{0}\right|<K$. For any $\varphi$, there exists an $\varepsilon>0$ such that (2.2) admits a unique solution in the sector $S_{\varepsilon . \varphi}=\{t \in C|0<| t|<\varepsilon,| \arg t \mid<\varphi\}$ with the properties

$$
\begin{aligned}
& \left|A_{\mu}(t)-A_{\mu}^{0}\right| \leq K|t|^{1-\sigma_{1}}, \quad\left|t^{-\Lambda}\left(A_{\mu}(t)-A_{\mu}^{0}\right) t^{\Lambda}\right| \leqq K^{2}|t|^{1-\sigma_{1}}, \\
& \left|t^{-\Lambda} B_{v}(t) t^{\Lambda}-\widetilde{B}_{v}^{0}\right| \leqq K|t|^{1-\sigma_{1}}
\end{aligned}
$$

(ii) Let $Y(x, t)$ be the corresponding solution of (2.1) normalized as

$$
Y(x, t)=\left(1+O\left(x^{-1}\right)\right) x^{-T_{0}^{(\infty)}} .
$$

Then the limits $Y(x)=\lim _{t \rightarrow 0} Y(x, t), \tilde{Y}(x)=\lim _{t \rightarrow 0} t^{-\Lambda} Y(t x, t)$ exist and satisfy the differential equations

$$
\begin{aligned}
& \frac{d Y}{d x}=\left(\sum_{\mu=1}^{n_{1}} \frac{A_{\mu}^{0}}{x-a_{\mu}}+\frac{\Lambda}{x}\right) Y \\
& \frac{d \tilde{Y}}{d x}=\left(\sum_{\nu=1}^{n_{2}} \frac{\tilde{B}_{v}^{0}}{x-b_{v}}\right) \tilde{Y}
\end{aligned}
$$

respectively.

We shall study the relation between the monodromy data for (2.1) and those for the limiting equations (2.5), (2.6). Let $Y_{0}(x)$ be the solution of (2.5) normalized at $x=\infty$. Taking into account the assumption (2.3), we see that its local behavior reads as follows:

$$
\begin{aligned}
Y_{0}(x) & =G_{0}^{(\mu)}\left(1+O\left(x-a_{\mu}\right)\right)\left(x-a_{\mu}\right)^{T_{0}^{(\mu)}} C^{(\mu)} & & \left(x \rightarrow a_{\mu}\right) \\
& =(1+O(x)) x^{\Lambda} C & & (x \rightarrow 0) \\
& =\left(1+O\left(x^{-1}\right)\right) x^{-T_{0}^{(\infty)}} & & (x \rightarrow \infty) .
\end{aligned}
$$

Here $G_{0}^{(\mu)}, C^{(\mu)}$ and $C$ are invertible, and $T_{0}^{(\mu)}$ are diagonal. Likewise, let $\tilde{Y}_{0}(t)$ be the solution of (2.6) such that

$$
\begin{aligned}
\tilde{Y}_{0}(x) & =\tilde{G}_{0}^{(v)}\left(1+O\left(x-b_{v}\right)\right)\left(x-b_{v}\right)^{\tilde{T}_{0}^{(\nu)}} \widetilde{C}^{(v)} & & \left(x \rightarrow b_{v}\right) \\
& =\left(1+O\left(x^{-1}\right)\right) x^{\Lambda} & & (x \rightarrow 0)
\end{aligned}
$$

with some $\widetilde{G}_{0}^{(v)}, \widetilde{C}^{(v)}$ and $\widetilde{T}_{0}^{(v)}$.

\section{Proposition 2.1. We have}

$$
\lim _{t \rightarrow 0} Y(x, t)=Y_{0}(x), \lim _{t \rightarrow 0} t^{-\Lambda} Y(t x, t)=\tilde{Y}_{0}(x) C .
$$

For $t \neq 0$, the local behavior of $Y(x, t)$ is given by 


$$
\begin{aligned}
Y(x, t) & =G^{(\mu)}(t)\left(1+O\left(x-a_{\mu}\right)\right)\left(x-a_{\mu}\right)^{T_{0}^{(\mu)}} C^{(\mu)} & & \left(x \rightarrow a_{\mu}\right) \\
& =\widetilde{G}^{(v)}(t)\left(1+O\left(x-t b_{v}\right)\right)\left(x-t b_{v}\right)^{\tilde{T}_{0}^{(v)}} \tilde{C}^{(v)} C & & \left(x \rightarrow t b_{v}\right)
\end{aligned}
$$

Here $G^{(\mu)}(t), \widetilde{G}^{(v)}(t)$ are invertible matrices, and $C^{(\mu)}, \widetilde{C}^{(v)}, C$ are given in (2.7), (2.8).

Proof. Let $F(x, t)=-\sum_{v=1}^{n_{2}} \frac{b_{v} B_{v}(t)}{x-t b_{v}}$, and set

$$
U(x, t)=1+\sum_{k=1}^{\infty} \int_{0}^{t} d t_{1} \int_{0}^{t_{1}} d t_{2} \cdots \int_{0}^{t_{k-1}} d t_{k} F\left(x, t_{1}\right) F\left(x, t_{2}\right) \cdots F\left(x, t_{k}\right) .
$$

The integration is taken along the line segment joining 0 and 1 in $S_{\varepsilon, \varphi}$. Thanks to (2.4), this converges uniformly with respect to $x$ on every compact subset of $\{x \in \mathbb{C}|| x|>| t \mid b\}, b=\max _{v}\left|b_{v}\right|$. By using (2.1), it is easy to establish

$$
Y(x, t)=U(x, t) Y_{0}(x), \quad|x|>|t| b .
$$

This proves the first half of the assertions (2.9), (2.10). Setting $\tilde{Y}(x, t)=t^{-\Lambda} Y(t x, t)$ and $\tilde{Y}_{0}^{\prime}(x)=\lim _{t \rightarrow 0} \tilde{Y}(x, t)$, we have likewise

$$
\tilde{Y}(x, t)=V(x, t) \tilde{Y}_{0}^{\prime}(x), \quad|x|<\frac{1}{|t|} a,
$$

where $a=\min _{v}\left|a_{v}\right|$, and $V(x, t)$ is a similar series with $\tilde{F}(x, t)=\frac{1}{t} \sum_{v=1}^{n_{2}}\left(t^{-\Lambda} B_{v}(t) t^{\Lambda}\right.$ $-\Lambda)+\sum_{\mu=1}^{n_{1}} \frac{x}{t x-a_{\mu}} t^{-\Lambda} A_{\mu}(t) t^{\Lambda}$ in place of $F(x, t)$. Proposition 2.1 is proved if we can show $\tilde{Y}_{0}^{\prime}(x)=\tilde{Y}_{0}(x) C$. Since $\tilde{Y}_{0}^{\prime}(x)$ solves $(2.6)$, it is sufficient to know its behavior at $x=\infty$. For this purpose we compare $\tilde{Y}(x, t)$ with $\tilde{Y}_{0}(x, t)$ $=t^{-\Lambda} Y_{0}(t x)$. Set

$$
X(x, t)=\tilde{Y}(x, t) \tilde{Y}_{0}(x, t)^{-1}=t^{\Lambda} U(t x, t) t^{-\Lambda} .
$$

For $t \neq 0$, it is single-valued holomorphic in $|x|>b$ with its value 1 at $x=\infty$. Moreover $X_{0}(x)=\lim _{t \rightarrow 0} X(x, t)$ exists uniformly on every compact subset of $|x|>b$. Hence $X_{0}(x)$ is also holomorphic at $x=\infty$, and has the leading behavior $1+O\left(x^{-1}\right)$. On the other hand, (2.3) and (2.7) imply

$$
\begin{aligned}
\lim _{t \rightarrow 0} \tilde{Y}_{0}(x, t) & =\lim _{t \rightarrow 0} t^{-\Lambda}\left(1+Y_{0,1} t x+\cdots\right)(t x)^{\Lambda} C \\
& =x^{\Lambda} C .
\end{aligned}
$$

This proves

$$
\tilde{Y}_{0}^{\prime}(x)=X_{0}(x) x^{\Lambda} C=\left(1+O\left(x^{-1}\right)\right) x^{\Lambda} C,
$$


thereby completing the proof of Proposition 2.1.

We note that, given the connection matrices $C^{(\mu)}, \widetilde{C}^{(\mu)} C$, the Riemann problem (2.10) has a solution $Y(x, t)$ for small $t$ and the coefficients $A_{\mu}(t)$, $B_{v}(t)$ necessarily behave like (2.4), provided the Riemann problems (2.7), (2.8) admit solutions with some $C$. This follows from the uniqueness of $Y(x, t)$.

Now we apply the results above to the present case of PVI, where both of the Riemann problems (2.7), (2.8) can be solved in terms of hypergeometric functions $F(\alpha, \beta, \gamma ; x)$.

Let us summarize the classical results. Consider the Riemann problem

$$
\begin{aligned}
Y(x) & =G_{0}(1+O(x)) x^{L^{(0)}} & & (x \rightarrow 0) \\
& =G_{1}(1+O(x-1))(x-1)^{L^{(1)}} & & (x \rightarrow 1) \\
& =\left(1+O\left(x^{-1}\right)\right) x^{-\left({ }_{\beta}\right)} & & (x \rightarrow \infty),
\end{aligned}
$$

the eigenvalues of $L^{(0)}=1-\gamma, 0$

the eigenvalues of $L^{(1)}=\gamma-\alpha-\beta-1,0$.

with the assumption

$$
1-\gamma, \gamma-\alpha-\beta-1 \notin \boldsymbol{Z}-\{0\}, \alpha-\beta \notin \boldsymbol{Z} .
$$

The monodromy matrices $M^{(v)}=e^{2 \pi i L^{(v)}}(v=0,1)$ satisfy the constraint $M^{(1)} M^{(0)}$ $=\left(\begin{array}{c}e^{-2 \pi i \alpha} \\ e^{-2 \pi i \beta}\end{array}\right)$, which can be solved as

$$
\begin{aligned}
& M^{(1)}=\frac{e^{\pi i(\gamma-\alpha-\beta)}}{i \sin \pi(\alpha-\beta)}\left(\begin{array}{cc}
\cos \pi \gamma-e^{-\pi i(\alpha-\beta)} \cos \pi(\gamma-\alpha-\beta) & p \\
q & -\cos \pi \gamma+e^{\pi i(\alpha-\beta)} \cos \pi(\gamma-\alpha-\beta)
\end{array}\right) \\
& M^{(0)}=\frac{e^{-\pi i \gamma}}{i \sin \pi(\alpha-\beta)}\left(\begin{array}{cc}
\cos \pi(\gamma-\alpha-\beta)-e^{-\pi i(\alpha-\beta)} & -e^{\pi i(\alpha-\beta)} p \\
-e^{-\pi i(\alpha-\beta)} q & -\cos \pi(\gamma-\alpha-\beta)+e^{\pi i(\alpha-\beta)} \cos \pi \gamma
\end{array}\right) \\
& p q=4 \sin \pi \alpha \sin \pi \beta \sin \pi(\gamma-\alpha) \sin \pi(\gamma-\beta) .
\end{aligned}
$$

In order that (2.11) has a solution, it is necessary and sufficient to have the following conditions.

(i) $p=0$ if one of $\alpha, \alpha-\gamma+1,-\beta,-(\beta-\gamma+1)$ is a positive integer

(ii) $q=0$ if one of $-\alpha,-(\alpha-\gamma+1), \beta, \beta-\gamma+1$ is a positive integer. Set 
(2.12) $Y(x ; \alpha, \beta, \gamma)$

$$
=\left(\begin{array}{r}
F\left(\alpha, \alpha-\gamma+1, \alpha-\beta ; \frac{1}{x}\right), \frac{\beta(\beta-\gamma+1)}{(\beta-\alpha)(\beta-\alpha+1)} \frac{1}{x} \\
\times F\left(\beta+1, \beta-\gamma+2, \beta-\alpha+2 ; \frac{1}{x}\right) \\
\frac{\alpha(\alpha-\gamma+1)}{(\alpha-\beta)(\alpha-\beta+1)} \frac{1}{x} F\left(\alpha+1, \alpha-\gamma+2, \alpha-\beta+2 ; \frac{1}{x}\right) \\
F\left(\beta, \beta-\gamma+1, \beta-\alpha ; \frac{1}{x}\right)
\end{array}\right) x^{-\left({ }_{\beta}{ }_{\beta}\right)} .
$$

If $\alpha \beta(\alpha-\gamma+1)(\beta-\gamma+1) \neq 0$, then $\left(\begin{array}{c}1 \\ r\end{array}\right)-1 Y(x ; \alpha, \beta, \gamma)\left(\begin{array}{c}1 \\ r\end{array}\right)$ gives the solution for some $r \neq 0$. If $\alpha \beta(\alpha-\gamma+1)(\beta-\gamma+1)=0$, then either of the following solves (2.11): $\left(\begin{array}{c}1 \\ r\end{array}\right)^{-1} Y(x ; \alpha, \beta, \gamma)\left(\begin{array}{c}1 \\ r\end{array}\right)$,

$$
\begin{gathered}
\left(\begin{array}{l}
1 \\
r
\end{array}\right)^{-1}\left(\begin{array}{c}
\alpha(\alpha-\gamma+1) \\
\beta(\beta-\gamma+1)
\end{array}\right) Y(x ; \alpha, \beta, \gamma)\left(\begin{array}{r}
\alpha(\alpha-\gamma+1) \\
\beta(\beta-\gamma+1)
\end{array}\right)^{-1}\left(\begin{array}{r}
1 \\
r
\end{array}\right), \\
\left(\begin{array}{rr}
1 & \\
x^{1-\gamma}(x-1)^{\gamma-\beta-1}
\end{array}\right),\left(\begin{array}{r}
x^{1-\gamma}(x-1)^{\gamma-\alpha-1} \\
1
\end{array}\right), \text { or }\left(\begin{array}{r}
x^{-\alpha} \\
(x-1)^{-\beta}
\end{array}\right) .
\end{gathered}
$$

For reference we give below the local behavior and the differential equation for $Y(x ; \alpha, \beta, \gamma)$.

$$
\begin{aligned}
& Y(x ; \alpha, \beta, \gamma)=G_{\alpha \beta \gamma}^{(0)}(1+O(x)) x^{\left(1-\gamma_{0}\right)} C_{\alpha \beta \gamma}^{(0)} \quad(x \rightarrow 0) \\
& \left.=G_{\alpha \beta \gamma}^{(1)}(1+O(x-1))(x-1)^{(\gamma-\alpha-\beta-1}\right)_{0} C_{\alpha \beta \gamma}^{(1)} \quad(x \rightarrow 1) \\
& =\left(1+O\left(x^{-1}\right)\right) x^{-\left(\begin{array}{c}
\alpha \\
\beta
\end{array}\right)} \quad(x \rightarrow \infty)
\end{aligned}
$$

where

$$
\begin{aligned}
& G_{\alpha \beta \gamma}^{(0)}=\frac{1}{\beta-\alpha}\left(\begin{array}{ll}
\beta-\gamma+1 & \beta \\
\alpha-\gamma+1 & \alpha
\end{array}\right), \quad G_{\alpha \beta \gamma}^{(1)}=\frac{1}{\beta-\alpha}\left(\begin{array}{ll}
1 & \beta(\beta-\gamma+1) \\
1 & \alpha(\alpha-\gamma+1)
\end{array}\right) \\
& C_{\alpha \beta \gamma}^{(0)}=\left(\begin{array}{cc}
e^{-\pi i(\alpha-\gamma+1)} \frac{\Gamma(\gamma-1) \Gamma(\alpha-\beta+1)}{\Gamma(\gamma-\beta) \Gamma(\alpha)} & -e^{-\pi i(\beta-\gamma+1)} \frac{\Gamma(\gamma-1) \Gamma(\beta-\alpha+1)}{\Gamma(\gamma-\alpha) \Gamma(\beta)} \\
e^{-\pi i \alpha} \frac{\Gamma(1-\gamma) \Gamma(\alpha-\beta+1)}{\Gamma(1-\beta) \Gamma(\alpha-\gamma+1)} & -e^{-\pi i \beta} \frac{\Gamma(1-\gamma) \Gamma(\beta-\alpha+1)}{\Gamma(1-\alpha) \Gamma(\beta-\gamma+1)}
\end{array}\right) \\
& C_{\alpha \beta \gamma}^{(1)}=\left(\begin{array}{cc}
-\frac{\Gamma(\alpha+\beta-\gamma+1) \Gamma(\alpha-\beta+1)}{\Gamma(\alpha-\gamma+1) \Gamma(\alpha)} & \frac{\Gamma(\alpha+\beta-\gamma+1) \Gamma(\beta-\alpha+1)}{\Gamma(\beta-\gamma+1) \Gamma(\beta)} \\
-e^{-\pi i(\gamma-\alpha-\beta-1)} \frac{\Gamma(\gamma-\alpha-\beta-1) \Gamma(\alpha-\beta+1)}{\Gamma(1-\beta) \Gamma(\gamma-\beta)} \\
e^{-\pi i(\gamma-\alpha-\beta-1)} \frac{\Gamma(\gamma-\alpha-\beta-1) \Gamma(\beta-\alpha+1)}{\Gamma(1-\alpha) \Gamma(\gamma-\alpha)}
\end{array}\right)
\end{aligned}
$$




$$
\begin{aligned}
\frac{d Y}{d x}= & {\left[\frac{1}{x} \frac{1}{\beta-\alpha}\left(\begin{array}{c}
\beta-\gamma+1 \\
\alpha-\gamma+1
\end{array}\right)(-\alpha \beta)\right.} \\
& \left.+\frac{1}{x-1} \frac{1}{\beta-\alpha}\left(\begin{array}{l}
1 \\
1
\end{array}\right)(\alpha(\alpha-\gamma+1),-\beta(\beta-\gamma+1))\right] Y .
\end{aligned}
$$

Returning to the situation (1.4), we find that

$$
\begin{aligned}
\lim _{t \rightarrow 0} Y(x, t)=\left(\begin{array}{c}
1 \\
r
\end{array}\right)^{-1} Y\left(x ; \frac{1}{2}\left(\theta_{\infty}-\theta_{1}-\sigma\right), \frac{1}{2}\right. & \left.\left(-\theta_{\infty}-\theta_{1}-\sigma\right), 1-\sigma\right) \\
& \times\left(\begin{array}{c}
1 \\
r
\end{array}\right) x^{-\sigma / 2}(x-1)^{-\theta_{1} / 2}
\end{aligned}
$$

with some $r \neq 0$. Without loss of generality we may take the overall parameter $r$ to be $1 . \quad Y_{0}(x)=\lim _{t \rightarrow 0} Y(x, t)$ satisfies

$$
\begin{aligned}
& \frac{d Y_{0}}{d x}=\left(\frac{\Lambda}{x}+\frac{A_{1}^{0}}{x-1}\right) Y_{0} \\
& \Lambda+\frac{1}{2} \sigma I_{2}=\frac{1}{4 \theta_{\infty}}\left(\begin{array}{c}
-\theta_{\infty}-\theta_{1}+\sigma \\
\theta_{\infty}-\theta_{1}+\sigma
\end{array}\right)\left(\theta_{\infty}-\theta_{1}-\sigma, \theta_{\infty}+\theta_{1}+\sigma\right), \\
& A_{1}^{0}+\frac{1}{2} \theta_{1} I_{2}=\frac{1}{4 \theta_{\infty}}\left(\begin{array}{c}
1 \\
1
\end{array}\right)\left(-\left(\theta_{\infty}-\theta_{1}\right)^{2}+\sigma^{2},\left(\theta_{\infty}+\theta_{1}\right)^{2}-\sigma^{2}\right) .
\end{aligned}
$$

We have also

$$
\begin{aligned}
& \lim _{t \rightarrow 0} t^{-\Lambda} Y(t x, t) \\
& =G_{1} Y\left(x ; \frac{1}{2}\left(-\theta_{0}-\theta_{t}-\sigma\right), \frac{1}{2}\left(-\theta_{0}-\theta_{t}+\sigma\right), 1-\theta_{0}\right) C_{1} x^{-\theta_{0} / 2}(x-1)^{-\theta_{t} / 2}
\end{aligned}
$$

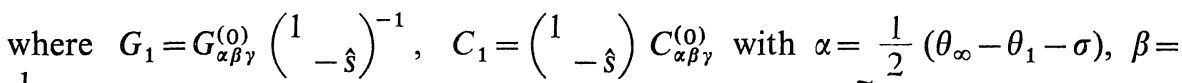
$\frac{1}{2}\left(-\theta_{\infty}-\theta_{1}-\sigma\right), \gamma=1-\sigma$, and $\hat{s}$ is given in (1.8). Hence $\tilde{Y}_{0}(x)=\lim _{t \rightarrow 0} t^{-\Lambda} Y(t x, t)$ satisfies

$$
\begin{aligned}
& \frac{d \tilde{Y}_{0}}{d x}=\left(\frac{A_{0}^{0}}{x}+\frac{A_{t}^{0}}{x-1}\right) \tilde{Y}_{0} \\
& A_{0}^{0}+\frac{1}{2} \theta_{0} I_{2}=G_{1} \frac{1}{4 \sigma}\left(\begin{array}{c}
\theta_{0}-\theta_{t}+\sigma \\
\theta_{0}-\theta_{t}-\sigma
\end{array}\right)\left(\theta_{0}+\theta_{t}+\sigma,-\theta_{0}-\theta_{t}+\sigma\right) G_{1}^{-1} \\
& A_{t}^{0}+\frac{1}{2} \theta_{t} I_{2}=G_{1} \frac{1}{4 \sigma}\left(\begin{array}{l}
1 \\
1
\end{array}\right)\left(\left(\theta_{t}+\sigma\right)^{2}-\theta_{0}^{2},-\left(\theta_{t}-\sigma\right)^{2}+\theta_{0}^{2}\right) G_{1}^{-1}
\end{aligned}
$$

From (2.4), (2.13) and (2.14), we obtain the following asymptotics of $A_{\mu}(t)$ :

(2.15) $A_{1}(t) \sim A_{1}^{0}$,

$$
A_{0}(t)+\frac{1}{2} \theta_{0} \cdot I_{2} \sim t^{\Lambda}\left(A_{0}^{0}+\frac{1}{2} \theta_{0} \cdot I_{2}\right) t^{-\Lambda}
$$




$$
\begin{aligned}
& =\frac{1}{16 \theta_{\infty} \sigma^{2}}\left(\begin{array}{l}
\left(\theta_{\infty}+\theta_{1}-\sigma\right)\left(\theta_{0}-\theta_{t}+\sigma\right) \\
-\left(\theta_{\infty}+\theta_{1}+\sigma\right)\left(\theta_{0}-\theta_{t}-\sigma\right) \hat{s}^{-1} \hat{\imath}^{-\sigma}, \\
\left(-\theta_{\infty}+\theta_{1}-\sigma\right)\left(\theta_{0}-\theta_{t}+\sigma\right) \\
-\left(-\theta_{\infty}+\theta_{1}+\sigma\right)\left(\theta_{0}-\theta_{t}-\sigma\right) \hat{s}^{-1} t^{-\sigma}
\end{array}\right) \\
& \times\left(\begin{array}{ll}
\left(\theta_{0}+\theta_{t}+\sigma\right)\left(-\theta_{\infty}+\theta_{1}+\sigma\right) & -\left(\theta_{0}+\theta_{t}+\sigma\right)\left(\theta_{\infty}+\theta_{1}+\sigma\right) \\
-\left(\theta_{0}+\theta_{t}-\sigma\right)\left(-\theta_{\infty}+\theta_{1}-\sigma\right) \hat{s} t^{\sigma}, & +\left(\theta_{0}+\theta_{t}-\sigma\right)\left(\theta_{\infty}+\theta_{1}-\sigma\right) \hat{s} t^{\sigma}
\end{array}\right) \\
& A_{t}(t)+\frac{1}{2} \theta_{t} \cdot I_{2} \sim t^{\Lambda}\left(A_{t}^{0}+\frac{1}{2} \theta_{t} \cdot I_{2}\right) t^{-\Lambda} \\
& =\frac{1}{16 \theta_{\infty} \sigma^{2}}\left(\begin{array}{l}
\left(\theta_{\infty}+\theta_{1}-\sigma\right) \\
-\left(\theta_{\infty}+\theta_{1}+\sigma\right) \hat{s}^{-1} t^{-\sigma} \\
\left(-\theta_{\infty}+\theta_{1}-\sigma\right) \\
-\left(-\theta_{\infty}+\theta_{1}+\sigma\right) \hat{s}^{-1} t^{-\sigma}
\end{array}\right) \\
& \times\left(\begin{array}{ll}
-\left(\left(\theta_{t}+\sigma\right)^{2}-\theta_{0}^{2}\right)\left(\theta_{\propto}-\theta_{1}-\sigma\right) & -\left(\left(\theta_{t}+\sigma\right)^{2}-\theta_{0}^{2}\right)\left(\theta_{\infty}+\theta_{1}+\sigma\right) \\
+\left(\left(\theta_{t}-\sigma\right)^{2}-\theta_{0}^{2}\right)\left(\theta_{\propto}-\theta_{1}+\sigma\right) \hat{s} t^{\sigma}, & +\left(\left(\theta_{t}-\sigma\right)^{2}-\theta_{0}^{2}\right)\left(\theta_{\infty}+\theta_{1}-\sigma\right) \hat{s} t^{\sigma}
\end{array}\right) .
\end{aligned}
$$

The case $\sigma=0$ can be incorporated if we set $\hat{s}=1+\sigma \hat{s}_{1}$ and let $\sigma \rightarrow 0$. From the definition (1.2) of the $\tau$ function and (2.15), using

$$
\frac{d}{d t}\left(t(\hat{t}-1) \frac{d}{d t} \log \tau(t)\right)=\theta_{\infty}\left(A_{t}(t)\right)_{22}-\frac{1}{2} \theta_{t}^{2}
$$

we obtain (1.9), (1.9)'.

Remark 1. The asymptotic expansion for the Painlevé transcendent $y(t)$ itself can be obtained by (2.15).

Remark 2. From the argument above, we see that the assumption (A.3) PvI $_{\mathrm{PV}}$ can be relaxed to include the following case.

If one of $\frac{1}{2}\left(\theta_{\infty} \pm \theta_{1} \pm^{\prime} \sigma\right)$ is a positive (resp. negative) integer, then $\left(M^{(1)}\right)_{12}$ $=0\left(\operatorname{resp} .\left(M^{(1)}\right)_{21}=0\right)$. If one of $\frac{1}{2}\left(\theta_{0} \pm \theta_{t} \pm^{\prime} \sigma\right)$ is a positive (resp. negative) integer, then $\left(C^{(0)} M^{(t)} C^{(0)-1}\right)_{12}=0$ (resp. $\left.\left(C^{(0)} M^{(t)} C^{(0)-1}\right)_{21}=0\right)$.

\section{§3. The Fifth and the Third Painlevé Equations}

By a similar reasoning as in the previous section, one can derive the short distance expansions for the fifth and the third Painleve equations.

First consider the case of PV, whose linear problem is 


$$
\begin{aligned}
& \frac{\partial Y}{\partial x}=\left(\frac{A_{0}(t)}{x}+\frac{A_{t}(t)}{x-t}+\left(\begin{array}{c}
1 \\
0
\end{array}\right)\right) Y, \\
& \frac{\partial Y}{\partial t}=-\frac{A_{t}(t)}{x-t} Y .
\end{aligned}
$$

We set

$$
\begin{aligned}
& \text { the eigenvalues of } A_{v}(t)= \pm \frac{1}{2} \theta_{v} \quad(v=0, t) \\
& \text { diagonal of } A_{0}(t)+A_{t}(t)=-\frac{1}{2}\left(\begin{array}{cc}
\theta_{\infty} & \\
& -\theta_{\infty}
\end{array}\right) .
\end{aligned}
$$

Then $y(t)=\left(A_{t}(t)\right)_{12}\left(\left(A_{0}(t)\right)_{11}+\frac{\theta_{0}}{2}\right) /\left(\left(A_{t}(t)\right)_{11}+\frac{\theta_{t}}{2}\right)\left(A_{0}(t)\right)_{12}$ satisfies (PV) with the parameters

$$
\begin{aligned}
& \alpha=\frac{1}{8}\left(\theta_{0}-\theta_{t}+\theta_{\infty}\right)^{2}, \quad \beta=-\frac{1}{8}\left(\theta_{0}-\theta_{t}-\theta_{\infty}\right)^{2}, \\
& \gamma=1-\theta_{0}-\theta_{t}, \quad \delta=-\frac{1}{2} .
\end{aligned}
$$

The $\tau$ function is defined by

$$
\frac{d}{d t} \log \tau(t)=\frac{1}{t}\left(A_{0}(t)+A_{t}(t)\right)_{12}\left(A_{0}(t)+A_{t}(t)\right)_{21}+\left(A_{t}(t)\right)_{11},
$$

and

$$
\zeta(t)=t \frac{d}{d t} \log \tau(t)+\frac{1}{2}\left(\theta_{0}+\theta_{\infty}\right) t+\frac{1}{4}\left(\left(\theta_{0}+\theta_{\infty}\right)^{2}-\theta_{t}^{2}\right)
$$

satisfies

$$
\begin{aligned}
& \left(t \frac{d^{2} \zeta}{d t^{2}}\right)^{2}=\left(\zeta-t \frac{d \zeta}{d t}+2\left(\frac{d \zeta}{d t}\right)^{2}-\left(2 \theta_{0}+\theta_{\infty}\right) \frac{d \zeta}{d t}\right)^{2} \\
& \quad-4 \frac{d \zeta}{d t}\left(\frac{d \zeta}{d t}-\theta_{0}\right)\left(\frac{d \zeta}{d t}-\frac{1}{2}\left(\theta_{0}-\theta_{t}+\theta_{\infty}\right)\right)\left(\frac{d \zeta}{d t}-\frac{1}{2}\left(\theta_{0}+\theta_{t}+\theta_{\infty}\right)\right)
\end{aligned}
$$

We assume

$(\mathrm{A} 1)_{\mathrm{PV}}$

$$
\theta_{0}, \theta_{t} \notin \mathbb{Z}
$$

Let $Y_{j}(x, t)$ be the solutions of (3.1) that have the normalized asymptotic behavior as $x \rightarrow \infty$ in the sector $\mathscr{S}_{j}=\left\{x \in \mathbf{C} \mid-\frac{\pi}{2}(2 j-1)<\arg x<\frac{\pi}{2}(-2 j+5)\right\}, j=1,2,3$. Namely:

$$
Y_{j}(x, t) \sim\left(1+O\left(x^{-1}\right)\right) x^{-\frac{1}{2}\left(\theta_{\infty}-\theta_{\infty}\right)} e^{\left(x_{0}\right),} \quad x \rightarrow \infty \quad \text { in } \mathscr{S}_{j} .
$$


They are related to each other by

$$
\begin{aligned}
& Y_{2}(x, t)=Y_{1}(x, t)\left(\begin{array}{ll}
1 & 0 \\
a & 1
\end{array}\right), \\
& Y_{3}(x, t) \equiv Y_{1}\left(x e^{2 \pi i}, t\right) e^{\pi i\left(\theta_{\infty}-\theta_{\infty}\right)}=Y_{2}(x, t)\left(\begin{array}{ll}
1 & b \\
0 & 1
\end{array}\right)
\end{aligned}
$$

with some constants $a, b \in \mathbb{C}$. At the regular singularities $x=0, t, Y_{1}(x, t)$ has the behavior

$$
\begin{aligned}
Y_{1}(x, t) & =G^{(0)}(t)(1+O(x)) x^{\frac{1}{2}\left({ }^{\theta_{0}}-\theta_{0}\right)} C^{(0)} & & (x \rightarrow 0) \\
& =G^{(t)}(t)(1+O(x-t))(x-t)^{\frac{1}{2}\left({ }^{\theta_{t}}-\theta_{t}\right)} C^{(t)} & & (x \rightarrow t) .
\end{aligned}
$$

The "Stokes multipliers" $a, b$ and the connection matrices $C^{(0)}, C^{(t)}$ constitute the monodromy data in the sense of [7]. They satisfy

$$
\begin{aligned}
& \left(\begin{array}{cc}
1 & b \\
a & 1+a b
\end{array}\right) e^{-\pi i\left(\theta_{\infty}-\theta_{\infty}\right)}=M^{(t)} M^{(0)}, \\
& M^{(v)}=C^{(v)-1}\left(\begin{array}{r}
e^{\pi i \theta_{v}} \\
e^{-\pi i \theta_{v}}
\end{array}\right) C^{(v)} \quad(v=0, t) .
\end{aligned}
$$

In order to parametrize them we set

$$
e^{\pi i \theta_{\infty}} a b+2 \cos \pi \theta_{\propto}=2 \cos \pi \sigma, \quad 0 \leqq \operatorname{Re} \sigma \leqq 1 .
$$

In the sequel we assume

$$
0 \leqq \operatorname{Re} \sigma \leqq 1
$$

$(\mathrm{A} 3)_{\mathrm{PV}}$

$$
\frac{1}{2}\left(\theta_{\infty} \pm \sigma\right), \frac{1}{2}\left(\theta_{t} \pm \theta_{0} \pm^{\prime} \sigma\right) \notin \mathbb{Z}
$$

Then the following parametrization is possible:

$\sigma \neq 0$

(3.7) $D^{(t)} C^{(t)} D=$ $\left(\begin{array}{c}\sin \frac{\pi}{2}\left(\theta_{t}+\theta_{0}+\sigma\right) \sin \frac{\pi}{2}\left(\theta_{t}-\theta_{0}+\sigma\right)-s \sin \frac{\pi}{2}\left(\theta_{t}+\theta_{0}-\sigma\right) \sin \frac{\pi}{2}\left(\theta_{t}-\theta_{0}-\sigma\right) \\ -s^{-1}\end{array}\right)$ $\times\left(\begin{array}{cc}e^{-\pi i \sigma / 2} & \sin \frac{\pi}{2}\left(\theta_{\infty}+\sigma\right) \\ e^{\pi i \sigma / 2} & \sin \frac{\pi}{2}\left(\theta_{\infty}-\sigma\right)\end{array}\right)$ 


$$
\begin{aligned}
& D^{(0)} C^{(0)} D \\
& =\left(\begin{array}{cc}
\sin \frac{\pi}{2}\left(\theta_{t}+\theta_{0}+\sigma\right) & -s e^{-\pi i \sigma} \sin \frac{\pi}{2}\left(\theta_{t}+\theta_{0}-\sigma\right) \\
-s^{-1} e^{\pi i \sigma} \sin \frac{\pi}{2}\left(\theta_{t}-\theta_{0}+\sigma\right) & \sin \frac{\pi}{2}\left(\theta_{t}-\theta_{0}-\sigma\right)
\end{array}\right) \\
& \times\left(\begin{array}{cc}
e^{-\pi i \sigma / 2} & \sin \frac{\pi}{2}\left(\theta_{\infty}+\sigma\right) \\
e^{\pi i \sigma / 2} & \sin \frac{\pi}{2}\left(\theta_{\infty}-\sigma\right)
\end{array}\right)
\end{aligned}
$$

$\sigma=0$

(3.7) $D^{(t)} C^{(t)} D$

$$
=\left(\begin{array}{ll}
\left(s_{1}+\pi i\right) \sin \frac{\pi}{2}\left(\theta_{t}+\theta_{0}\right) & \left(s_{1} \sin \frac{\pi}{2} \theta_{\infty}-\pi \cos \frac{\pi}{2} \theta_{\infty}\right) \sin \frac{\pi}{2}\left(\theta_{t}+\theta_{0}\right) \\
\times \sin \frac{\pi}{2}\left(\theta_{t}-\theta_{0}\right)-\pi \sin \pi \theta_{t} & \times \sin \frac{\pi}{2}\left(\theta_{t}-\theta_{0}\right)-\pi \sin \pi \theta_{t} \sin \frac{\pi}{2} \theta_{\infty} \\
s_{1}+\pi i & s_{1} \sin \frac{\pi}{2} \theta_{\infty}-\pi \cos \frac{\pi}{2} \theta_{\infty}
\end{array}\right)
$$

$D^{(0)} C^{(0)} D$

$$
=\left(\begin{array}{rr}
s_{1} \sin \frac{\pi}{2}\left(\theta_{t}+\theta_{0}\right)-\pi \cos \frac{\pi}{2}\left(\theta_{t}+\theta_{0}\right) & \left(s_{1}-\pi i\right) \sin \frac{\pi}{2}\left(\theta_{t}+\theta_{0}\right) \sin \frac{\pi}{2} \theta_{\infty} \\
s_{1} \sin \frac{\pi}{2}\left(\theta_{t}-\theta_{0}\right)-\pi \cos \frac{\pi}{2}\left(\theta_{t}-\theta_{0}\right) & -\sin \frac{\pi}{2}\left(\theta_{t}+\theta_{0}+\theta_{\infty}\right) \\
\left(s_{1}-\pi i\right) \sin \frac{\pi}{2}\left(\theta_{t}-\theta_{0}\right) \sin \frac{\pi}{2} \theta_{\infty} \\
-\sin \frac{\pi}{2}\left(\theta_{t}-\theta_{0}+\theta_{\infty}\right)
\end{array}\right) .
$$

Here $D^{(t)}, D^{(0)}$ and $D$ are invertible diagonal matrices.

Theorem 3.1. Under the parametrization (3.7)-(3.7)', we have, for $\sigma \neq 0$,

$$
\begin{aligned}
& \tau(t) \sim \text { const. } t^{\left(\sigma^{2}-\theta_{\infty}^{2}\right) / 4}\left[1-\frac{1}{4 \sigma^{2}} \theta_{\infty}\left(\theta_{t}^{2}-\theta_{0}^{2}+\sigma^{2}\right) t\right. \\
& +\frac{1}{8 \sigma^{2}(1+\sigma)^{2}} \hat{s}\left(\theta_{\infty}-\sigma\right)\left(\left(\theta_{t}-\sigma\right)^{2}-\theta_{0}^{2}\right) t^{1+\sigma} \\
& +\frac{1}{8 \sigma^{2}(1-\sigma)^{2}} \hat{s}^{-1}\left(\theta_{\infty}+\sigma\right)\left(\left(\theta_{t}+\sigma\right)^{2}-\theta_{0}^{2}\right) t^{1-\sigma} \\
& \left.+O\left(|t|^{2(1-\mathrm{Re} \sigma)}\right)\right] \text {, } \\
& \hat{s}=\frac{\Gamma\left(\frac{1}{2}\left(\theta_{t}+\theta_{0}+\sigma\right)+1\right) \Gamma\left(\frac{1}{2}\left(\theta_{t}-\theta_{0}+\sigma\right)+1\right) \Gamma\left(\frac{1}{2}\left(\theta_{\infty}+\sigma\right)+1\right) \Gamma(1-\sigma)^{2}}{\Gamma\left(\frac{1}{2}\left(\theta_{t}+\theta_{0}-\sigma\right)+1\right) \Gamma\left(\frac{1}{2}\left(\theta_{t}-\theta_{0}-\sigma\right)+1\right) \Gamma\left(\frac{1}{2}\left(\theta_{\infty}-\sigma\right)+1\right) \Gamma(1+\sigma)^{2}} s .
\end{aligned}
$$


If $\sigma=0$, then

$$
\begin{aligned}
\tau(t) \sim \text { const. } t^{-\theta_{\infty}^{2} / 4}[1 & +\frac{t}{2} \theta_{t} \\
& +\frac{1}{8} \theta_{\infty}\left(\theta_{t}^{2}-\theta_{0}^{2}\right) t\left(\Omega^{2}+2 \Omega+3\right) \\
& +-\frac{1}{4}\left(\theta_{t}^{2}-\theta_{0}^{2}+2 \theta_{\infty} \theta_{t}\right) t(\Omega+1) \\
& +o(t)]
\end{aligned}
$$

with $\Omega=1-\hat{s}_{1}-\log t$, and

$$
\hat{s}_{1}=s_{1}+\psi\left(\frac{1}{2}\left(\theta_{t}+\theta_{0}\right)+1\right)+\psi\left(\frac{1}{2}\left(\theta_{t}-\theta_{0}\right)+1\right)+\psi\left(\frac{1}{2} \theta_{\infty}+1\right)-4 \psi(1) .
$$

These formulas are derived in the same manner as in Section 2. We need also the confluent hypergeometric functions. Set

(3.10) $\quad Y_{\frac{1}{2}}(x ; \kappa, \sigma)$

$$
\begin{aligned}
&=\left(\begin{array}{cc}
e^{ \pm \pi i(1-\kappa) / 2} W_{(1-\kappa) / 2, \sigma / 2}\left(e^{\mp \pi i} x\right) & -\frac{1}{2}(\sigma-\kappa) W_{(-1+\kappa) / 2, \sigma / 2}(x) \\
-\frac{1}{2}(\sigma+\kappa) e^{ \pm \pi i(1-\kappa) / 2} W_{-(1+\kappa) / 2, \sigma / 2}\left(e^{\mp \pi i} x\right) & W_{(1+\kappa) / 2, \sigma / 2}(x)
\end{array}\right) \\
& \times x^{-1 / 2} e^{x / 2}
\end{aligned}
$$

where $W_{\kappa, \mu}(x)$ denotes the Whittaker function [11]. Then

$$
\begin{aligned}
& Y_{j}(x ; \kappa, \sigma) \sim\left(1+O\left(x^{-1}\right)\right)\left(\frac{1}{x}\right)^{\frac{1}{2}\left({ }^{\kappa}-\kappa\right)} e^{\left(x_{0}\right)} \quad\left(x \rightarrow \infty \text { in } \mathscr{S}_{j}\right) \\
& Y_{2}(x ; \kappa, \sigma)=Y_{1}(x ; \kappa, \sigma)\left(\begin{array}{cc}
1 & 0 \\
a_{\kappa \sigma} & 1
\end{array}\right), Y_{3}(x ; \kappa, \sigma)=Y_{1}(x ; \kappa, \sigma)\left(\begin{array}{cc}
1 & b_{\kappa \sigma} \\
0 & 1
\end{array}\right) \\
& Y_{1}(x ; \kappa, \sigma)=G_{\kappa \sigma}(1+O(x)) x^{\frac{1}{2}\left({ }^{\sigma}-\sigma\right)} C_{\kappa \sigma}
\end{aligned}
$$

with $\left.Y_{3}(x ; \kappa, \sigma)=Y_{1}\left(x e^{2 \pi i} ; \kappa, \sigma\right) e^{\pi i\left({ }^{\kappa}-\kappa\right.}\right)$, and

$$
\begin{gathered}
a_{\kappa \sigma}=2 \pi i \frac{1}{\Gamma\left(\frac{\sigma+\kappa}{2}\right) \Gamma\left(1-\frac{\sigma-\kappa}{2}\right)}, b_{\kappa \sigma}=2 \pi i e^{-\pi i \kappa} \frac{1}{\Gamma\left(1-\frac{\sigma+\kappa}{2}\right) \Gamma\left(\frac{\sigma-\kappa}{2}\right)} \\
G_{\kappa \sigma}=\left(\begin{array}{cc}
\frac{1}{2}(\sigma-\kappa) & -1 \\
\frac{1}{2}(\sigma+\kappa) & 1
\end{array}\right), C_{\kappa \sigma}=\left(\begin{array}{lc}
\frac{-\Gamma(-\sigma)}{\Gamma\left(1-\frac{\sigma-\kappa}{2}\right)} e^{-\pi i(\sigma+\kappa) / 2} \frac{-\Gamma(-\sigma)}{\Gamma\left(1-\frac{\sigma+\kappa}{2}\right)} \\
\frac{-\Gamma(\sigma)}{\Gamma\left(\frac{\sigma+\kappa}{2}\right)} e^{\pi i(\sigma-\kappa) / 2} & \frac{\Gamma(\sigma)}{\Gamma\left(\frac{\sigma-\kappa}{2}\right)}
\end{array}\right) .
\end{gathered}
$$


The differential equation for $Y_{j}(x ; \kappa, \sigma)$ reads

$$
\frac{d Y}{d x}=\left(\frac{1}{x} \frac{1}{2}\left(\begin{array}{cc}
-\kappa & \sigma-\kappa \\
\sigma+\kappa & \kappa
\end{array}\right)+\left(\begin{array}{ll}
1 & \\
& 0
\end{array}\right)\right) Y .
$$

We have then the following result.

$$
\lim _{t \rightarrow 0}\left(\begin{array}{ll}
1 & \\
& r
\end{array}\right) Y_{\frac{1}{2}}(x, t)\left(\begin{array}{ll}
1 & \\
& r
\end{array}\right)^{-1}=Y_{\frac{1}{2}}\left(x ; \theta_{\infty}, \sigma\right)
$$

where $\quad r^{-1}=\Gamma\left(\frac{\sigma+\theta_{\infty}}{2}\right) \Gamma\left(1-\frac{\sigma-\theta_{\infty}}{2}\right) a / 2 \pi i$,

$$
\begin{aligned}
\lim _{t \rightarrow 0} t^{-\Lambda}\left(\begin{array}{c}
1 \\
r
\end{array}\right) Y_{1}(t x, t)\left(\begin{array}{l}
1 \\
r
\end{array}\right)^{-1} \\
=G_{1} Y\left(x ; \frac{1}{2}\left(-\theta_{t}-\theta_{0}-\sigma\right), \frac{1}{2}\left(-\theta_{0}-\theta_{t}+\sigma\right), 1-\theta_{0}\right) C_{1},
\end{aligned}
$$

where $\Lambda=\frac{1}{2}\left(\begin{array}{cc}-\theta_{\infty} & \sigma-\theta_{\infty} \\ \sigma+\theta_{\infty} & \theta_{\infty}\end{array}\right)$ and $G_{1}=G_{\theta_{\infty} \sigma}\left(\begin{array}{c}1 \\ -\hat{s} \frac{\theta_{\infty}+\sigma}{2}\end{array}\right)^{-1}, C_{1}=\left(\begin{array}{c}1 \\ -\hat{s} \frac{\theta_{\infty}+\sigma}{2}\end{array}\right)$ $\times C_{\theta_{\infty} \sigma}$. For $\sigma=0$, one can take the limit $\sigma \rightarrow 0$ by setting $\hat{s}=1+\sigma \hat{s}_{1}$. The rest of the argument is the same, except that we use $\frac{d}{d t}\left(t \frac{d}{d t} \log \tau\right)=\left(A_{t}(t)\right)_{11}$ in place of (2.15). As a result we obtain

$$
\begin{aligned}
& A_{0}(t)+\frac{1}{2} \theta_{0} \cdot I_{2} \sim \frac{1}{8 \sigma^{2}}\left(\begin{array}{c}
-\left(\theta_{\infty}-\sigma\right)\left(-\theta_{t}+\theta_{0}+\sigma\right) \\
+\left(\theta_{\infty}+\sigma\right)\left(-\theta_{t}+\theta_{0}-\sigma\right) \hat{s}^{-1} t^{-\sigma}, \\
\left(\theta_{\infty}+\sigma\right)\left(\left(-\theta_{t}+\theta_{0}+\sigma\right)\right. \\
\left.-\left(-\theta_{t}+\theta_{0}-\sigma\right) \hat{s}^{-1} t^{-\sigma}\right)
\end{array}\right) \\
& \times\left(\begin{array}{cc}
\left(\theta_{t}+\theta_{0}+\sigma\right) & \left(\theta_{\infty}+\sigma\right)^{-1}\left(\left(\theta_{\infty}+\sigma\right)\left(\theta_{t}+\theta_{0}+\sigma\right)\right. \\
-\left(\theta_{t}+\theta_{0}-\sigma\right) \hat{s} t^{\sigma}, & \left.-\left(\theta_{\infty}-\sigma\right)\left(\theta_{t}+\theta_{0}-\sigma\right) \hat{s} t^{\sigma}\right)
\end{array}\right) \\
& A_{t}(t)+\frac{1}{2} \theta_{t} \cdot I_{2} \sim \frac{1}{8 \sigma^{2}}\left(\begin{array}{l}
-\left(\theta_{\infty}-\sigma\right) \\
+\left(\theta_{\infty}+\sigma\right) \hat{s}^{-1} t^{-\sigma}, \\
\left(\theta_{\infty}+\sigma\right)\left(1-\hat{s}^{-1} t^{-\sigma}\right)
\end{array}\right) \\
& \times\left(\begin{array}{cc}
\left(\theta_{t}+\sigma\right)^{2}-\theta_{0}^{2} & \left(\theta_{\infty}+\sigma\right)^{-1}\left(\left(\theta_{\infty}+\sigma\right)\left(\left(\theta_{t}+\sigma\right)^{2}-\theta_{0}^{2}\right)\right. \\
-\left(\left(\theta_{t}-\sigma\right)^{2}-\theta_{0}^{2}\right) \hat{s} t^{\sigma}, & \left.-\left(\theta_{\infty}-\sigma\right)\left(\left(\theta_{t}-\sigma\right)^{2}-\theta_{0}^{2}\right) \hat{s} t^{\sigma}\right)
\end{array}\right)
\end{aligned}
$$

from which the behavior of the fifth Painleve transcendent $y(t)$ follows.

Now we proceed to PIII. Consider the linear differential equation

$$
\begin{aligned}
& \frac{\partial Y}{\partial x}=\left(\frac{-t A(t)}{x^{2}}+\frac{B(t)}{x}+\left(\begin{array}{ll}
1 & \\
& 0
\end{array}\right)\right) Y, A(t)=G(t)\left(\begin{array}{ll}
1 & \\
& 0
\end{array}\right) G(t)^{-1} \\
& \frac{\partial Y}{\partial t}=\frac{A(t)}{x} Y .
\end{aligned}
$$


Let $\widetilde{B}(t)=G(t)^{-1} B(t) G(t)$, and set

$$
\begin{aligned}
& \text { diagonal of } B(t)=\frac{-1}{2}\left(\begin{array}{ll}
\theta_{\infty} & \\
& -\theta_{\infty}
\end{array}\right) \\
& \text { diagonal of } \widetilde{B}(t)=\frac{1}{2}\left(\begin{array}{cc}
\theta_{0} & \\
& -\theta_{0}
\end{array}\right) .
\end{aligned}
$$

Then $y(t)=-B(t)_{12} / \sqrt{t} A(t)_{12}$ satisfies (PIII) with respect to the variable $\bar{t}=\sqrt{t}$, where

$$
\alpha=4 \theta_{0}, \quad \beta=4\left(1-\theta_{\infty}\right), \quad \gamma=4, \quad \delta=-4 .
$$

Define the $\tau$ function by

$$
\frac{d}{d t} \log \tau(t)=\frac{1}{t} \widetilde{B}(t)_{12} \widetilde{B}(t)_{21}-A(t)_{11} .
$$

Setting

$$
\zeta(t)=t \frac{d}{d t} \log \tau(t)+\frac{1}{4}\left(\theta_{0}^{2}-\theta_{\infty}^{2}\right)+t
$$

we have

$$
\left(t \frac{d^{2} \zeta}{d t^{2}}\right)^{2}=4 \frac{d \zeta}{d t}\left(\frac{d \zeta}{d t}-1\right)\left(\zeta-t \frac{d \zeta}{d t}\right)+\left(\theta_{\infty} \frac{d \zeta}{d t}-\frac{\theta_{0}+\theta_{\infty}}{2}\right)^{2}
$$

The monodromy data for (3.9) are introduced in the following way. At $x=\infty$, there exist unique solutions $Y_{j}(x, t)(j=1,2,3)$ such that

$$
\begin{gathered}
Y_{j}(x, t) \sim\left(1+O\left(x^{-1}\right)\right) x^{-\frac{1}{2}\left(\theta_{\infty}-\theta_{\infty}\right)} e^{\left(x_{0}\right)}, \quad x \rightarrow \infty \quad \text { in } \mathscr{S}_{j} . \\
Y_{2}(x, t)=Y_{1}(x, t)\left(\begin{array}{ll}
1 & 0 \\
a & 1
\end{array}\right) \\
Y_{3}(x, t) \equiv Y_{1}\left(x e^{2 \pi i}, t\right) e^{\pi i\left(\theta_{\infty}-\theta_{\infty}\right)}=Y_{2}(x, t)\left(\begin{array}{ll}
1 & b \\
0 & 1
\end{array}\right)
\end{gathered}
$$

where $\mathscr{S}_{j}=\left\{x \in \mathbb{C} \mid-\frac{\pi}{2}(2 j-1)<\arg x<-\frac{\pi}{2}(2 j-5)\right\}$. Likewise, at $x=0$, there are solutions $\bar{Y}_{j}(x, t)(j=1,2,3)$ with the properties

$$
\begin{aligned}
& \bar{Y}_{j}(x, t) \sim G(t)(1+O(x)) x^{\frac{1}{2}\left(\theta_{0}-\theta_{0}\right)} e^{\left(t_{0}^{t}\right) \frac{1}{x},} x \rightarrow 0 \text { in } \overline{\mathscr{S}}_{j}(t) \\
& \bar{Y}_{2}(x, t)=\bar{Y}_{1}(x, t)\left(\begin{array}{ll}
1 & 0 \\
\bar{a} & 1
\end{array}\right)
\end{aligned}
$$




$$
\bar{Y}_{3}(x, t) \equiv \bar{Y}_{1}\left(x e^{-2 \pi i}, t\right) e^{\pi i\left(\theta_{0}-\theta_{0}\right)}=\bar{Y}_{2}(x, t)\left(\begin{array}{ll}
1 & \bar{b} \\
0 & 1
\end{array}\right)
$$

where $\overline{\mathscr{S}}_{j}(t)=\left\{x \in \mathbb{C} \mid-\frac{\pi}{2}(2 j-1)<\arg \left(\frac{t}{x}\right)<-\frac{\pi}{2}(2 j-5)\right\}$. We fix a path $\gamma$ joining $\infty$ and 0 so that $x \in \mathscr{S}_{1}$ (resp. $x \in \overline{\mathscr{S}}_{2}(t)$ ) as $x \rightarrow \infty$ (resp. $x \rightarrow 0$ ) along $\gamma$, and define the connection matrix $C$ by

$$
Y_{1}(x, t)=\bar{Y}_{2}(x, t) C .
$$

Here both sides signify their branches on $\gamma$. The monodromy data for (3.9) are $a, b, \bar{a}, \bar{b}$ and $C$. They are related through

$$
\left(\begin{array}{cc}
1 & b \\
a & 1+a b
\end{array}\right) e^{-\pi i\left(\theta_{\infty}-\theta_{\infty}\right)}=C^{-1}\left(\begin{array}{rr}
1 & 0 \\
-\bar{a} & 1
\end{array}\right) e^{\pi i\left(\theta_{0}-\theta_{0}\right)}\left(\begin{array}{rr}
1 & -\bar{b} \\
0 & 1
\end{array}\right) C .
$$

Let us introduce $\sigma \in \mathbb{C}$ by

(3.18) $e^{\pi i \theta_{\infty}} a b+2 \cos \pi \theta_{\infty}=e^{\pi i \theta_{0}} \bar{a} \bar{b}+2 \cos \pi \theta_{0}=2 \cos \pi \sigma$

$$
0 \leqq \operatorname{Re} \sigma \leqq 1 \text {, }
$$

the first equality being a consequence of (3.17). Again we assume

$(\mathrm{A} .1)_{\mathrm{PIII}}$

$$
0 \leqq \operatorname{Re} \sigma<1
$$

$(\mathrm{A} .2)_{\mathrm{PIII}}$

$$
\frac{1}{2}\left(\theta_{\infty} \pm \sigma\right), \frac{1}{2}\left(\theta_{0} \pm \sigma\right) \notin \mathbb{Z} \text {. }
$$

Then we can parametrize $C$ as follows:

$$
\begin{aligned}
& \underline{\sigma \neq 0} \quad C=k\left(-\frac{\bar{b} e^{\pi i \theta_{0}}}{2 i s}\right)^{-1} \\
& \times\left(\begin{array}{cc}
1 & -s \\
-s^{-1} e^{\pi i\left(\sigma+\theta_{0}\right) / 2} \sin \frac{\pi}{2}\left(-\sigma+\theta_{0}\right) & e^{\pi i\left(-\sigma+\theta_{0}\right) / 2} \sin \frac{\pi}{2}\left(\sigma+\theta_{0}\right)
\end{array}\right) \\
& \times\left(\begin{array}{ll}
e^{-\pi i\left(\sigma+\theta_{\infty}\right) / 2} \sin \frac{\pi}{2}\left(-\sigma+\theta_{\infty}\right) & 1 \\
e^{\pi i\left(\sigma-\theta_{\infty}\right) / 2} \sin \frac{\pi}{2}\left(\sigma+\theta_{\infty}\right) & 1
\end{array}\right)\left(\begin{array}{l}
1 \\
-\frac{b e^{\pi i \theta_{0}}}{2 i}
\end{array}\right)
\end{aligned}
$$

where $k, s$ are nonzero constants, 
$\frac{\sigma=0}{(3.19)^{\prime}}, \quad C=k\left(-\frac{1}{2 i} e^{\pi i \theta_{0}}\right)^{-1}$

$$
\begin{aligned}
& \times\left(\begin{array}{lc}
-s_{1} e^{-\pi i \theta_{\infty} / 2} \sin \frac{\pi}{2} \theta_{r}-\pi & -s_{1} \\
e^{\pi i\left(\theta_{0}-\theta_{a}\right) / 2}\left(s_{1} \sin \frac{\pi \theta_{0}}{2} \sin \frac{\pi \theta_{\infty}}{2}+\pi \sin \frac{\pi}{2}\left(\theta_{0}+\theta_{\infty}\right)\right) s_{1} e^{\pi i \theta_{0} / 2} \sin \frac{\pi}{2} \theta_{0}+\pi
\end{array}\right) \\
& \times\left(\begin{array}{ll}
1 & \\
& -\frac{b e^{\pi i \theta_{0}}}{2 i}
\end{array}\right) \text {. }
\end{aligned}
$$

Theorem 3.2. Under the assumptions (A.1) $)_{\mathrm{PIII}}-(\mathrm{A} .2)_{\mathrm{PIII}}$ and the parametrization (3.19)-(3.19)', the following asymptotic expansions are valid. $\underline{\sigma \neq 0} \tau(t) \sim$ const. $t^{\left(\sigma^{2}-\theta_{0}^{2}\right) / 4}\left(1+\frac{1}{2 \sigma^{2}}\left(\theta_{0} \theta_{\infty}-\sigma^{2}\right) t-\frac{\left(\theta_{0}+\sigma\right)\left(\theta_{\infty}+\sigma\right)}{4 \sigma^{2}(1+\sigma)^{2}} \hat{s} t^{1+\sigma}\right.$

$$
\left.-\frac{\left(\theta_{0}-\sigma\right)\left(\theta_{\infty}-\sigma\right)}{4 \sigma^{2}(1-\sigma)^{2}} \hat{s}^{-1} t^{1-\sigma}+O\left(|t|^{2(1-\operatorname{Re} \sigma)}\right)\right)
$$

where

$$
\hat{s}=\frac{\Gamma(1-\sigma)^{2} \Gamma\left(1+\frac{\sigma-\theta_{0}}{2}\right) \Gamma\left(1+\frac{\sigma-\theta_{\infty}}{2}\right)}{\Gamma(1+\sigma)^{2} \Gamma\left(1-\frac{\sigma+\theta_{0}}{2}\right) \Gamma\left(1-\frac{\sigma+\theta_{\infty}}{2}\right)} s .
$$

$\underline{\sigma=0} \tau(t) \sim$ const. $t^{-\theta_{0}^{2} / 4}\left(1-t+\frac{1}{2}\left(\theta_{0}+\theta_{\infty}\right) t(\Omega+1)-\frac{1}{4} \theta_{0} \theta_{\infty} t\left(\Omega^{2}+2 \Omega+3\right)\right.$

$$
+o(t))
$$

where $\Omega=1-\hat{s}_{1}-\log t$, and

$$
\hat{s}_{1}=s_{1}+\psi\left(1-\frac{\theta_{0}}{2}\right)+\psi\left(1-\frac{\theta_{\infty}}{2}\right)-4 \psi(1) .
$$

Example. As an application of the formulas above, let us consider the special case of PIII where the connection matrix $C$ is 1 . In view of (3.19)$(3.19)^{\prime}$, this happens if and only if $s=1$ (or $s_{1}=0$ ) and $\theta_{0}+\theta_{\alpha} \in 2 \mathbb{Z}$. Here we discuss the case

$$
\theta_{0}=-\theta_{\propto}=\theta .
$$

The general case of $\theta_{0}+\theta_{\infty} \in 2 \mathbb{Z}$ can be achieved by the Schlesinger transformation ([7] II).

It is known ([5] IV) that both the matrix $Y(x, 1)$ and the $\tau$ function allow convergent series expressions as follows. 


$$
\begin{aligned}
\left(Y_{1}(x, t)\right)_{11}= & \sum_{n=0}^{\infty} \lambda^{2 n} \int_{0}^{\infty} \cdots \int_{0}^{\infty} \prod_{j=1}^{2 n}\left(\frac{d x_{j}}{2 \pi} e^{-\left(x_{j}+t x_{j}^{-1}\right)}\right)\left(\frac{x_{1} x_{3} \cdots x_{2 n-1}}{x_{2} x_{4} \cdots x_{2 n}}\right)^{\theta} \\
& \times\left(\prod_{l=1}^{2 n-1} \frac{1}{x_{l}+x_{l+1}}\right) \frac{1}{x_{2 n}-x} x^{\theta / 2} e^{x+t x^{-1}} \\
\left(Y_{1}(x, t)\right)_{21}= & r \sum_{n=0}^{\infty} \lambda^{2 n+1} \int_{0}^{\infty} \cdots \int_{0}^{\infty} \prod_{j=1}^{2 n+1}\left(\frac{d x_{j}}{2 \pi} e^{-\left(x_{j}+t x_{\jmath}^{-1}\right)}\right)\left(\frac{x_{2} x_{4} \cdots x_{2 n}}{x_{1} x_{3} \cdots x_{2 n-1}} \frac{1}{x_{2 n+1}}\right)^{\theta} \\
& \times\left(\prod_{l=1}^{2 n} \frac{1}{x_{l}+x_{l+1}}\right) \frac{1}{x_{2 n+1}-x} x^{\theta / 2} e^{x+t x^{-1}} \\
\left(Y_{1}(x, t)\right)_{12}= & r^{-1} \sum_{n=0}^{\infty} \lambda^{2 n+1} \int_{0}^{\infty} \cdots \int_{0}^{\infty} \prod_{j=1}^{2 n+1}\left(\frac{d x_{j}}{2 \pi} e^{-\left(x_{j}+t x_{j}^{-1}\right)}\right) \\
& \times\left(\frac{x_{1} x_{3} \cdots x_{2 n-1}}{x_{2} x_{4} \cdots x_{2 n}} x_{2 n+1}\right)^{\theta}\left(\prod_{l=1}^{2 n} \frac{1}{x_{l}+x_{l+1}}\right) \frac{1}{x_{2 n+1}+x} x^{-\theta / 2} \\
\left(Y_{1}(x, t)\right)_{22}= & \sum_{n=0}^{\infty} \lambda^{2 n} \int_{0}^{\infty} \cdots \int_{0}^{\infty} \prod_{j=1}^{2 n}\left(\frac{d x_{j}}{2 \pi} e^{-\left(x_{j}+t x_{j}^{-1}\right)}\right)\left(\frac{x_{1} x_{3} \cdots x_{2 n-1}}{x_{2} x_{4} \cdots x_{2 n}}\right)^{\theta} \\
& \times\left(\prod_{l=1}^{2 n-1} \frac{1}{x_{l}+x_{l+1}}\right) \frac{1}{x_{2 n}+x} x^{-\theta / 2}
\end{aligned}
$$

(3.21) $e^{t} \tau(t)$

$$
\begin{aligned}
=\exp \left[-\sum_{n=1}^{\infty}\right. & \frac{\lambda^{2 n}}{n} \int_{0}^{\infty} \cdots \int_{0}^{\infty} \prod_{j=1}^{2 n}\left(\frac{d x_{j}}{2 \pi} e^{-\left(x_{j}+t x^{-1}\right)}\right)\left(\frac{x_{1} x_{3} \cdots x_{2 n-1}}{x_{2} x_{4} \cdots x_{2 n}}\right)^{\theta} \\
& \left.\times\left(\prod_{l=1}^{2 n-1} \frac{1}{x_{l}+x_{l+1}}\right) \frac{1}{x_{2 n}+x_{1}}\right] .
\end{aligned}
$$

In particular, (3.21) is related to the two point correlation function of the Federbush model ([5] Supplement to IV).

Let

$$
\begin{aligned}
& Y_{2}(x, t)=\left(\begin{array}{ll}
\left(Y_{1}\left(x e^{2 \pi i}, t\right)\right)_{11} & \left(Y_{1}(x, t)\right)_{12} \\
\left(Y_{1}\left(x e^{2 \pi i}, t\right)\right)_{21} & \left(Y_{1}(x, t)\right)_{22}
\end{array}\right) e^{-\pi i \theta\left({ }_{0}{ }_{0}\right)}, \\
& Y_{3}(x, t)=Y_{1}\left(x e^{2 \pi i}, t\right) e^{-\pi i \theta\left({ }^{1}{ }_{-1}\right)} .
\end{aligned}
$$

Then we have the relations (3.14) with

$$
a=i r \lambda, b=i r^{-1} \lambda e^{\pi i \theta} .
$$

The parametrization (3.18) reads

$$
\lambda^{2}=4 \sin \frac{\pi}{2}(\sigma+\theta) \sin \frac{\pi}{2}(\sigma-\theta) .
$$

Hence (3.20)-(3.20)' yield the following result.

Proposition 3.3. Let $\tau(t)$ be defined by the series (3.21). Assume that $\frac{1}{2} \lambda^{2}-\cos \pi \theta$ is not a real number $\geqq 1$, and let $\sigma$ be defined by (3.22), $0 \leqq$ $\operatorname{Re} \sigma<1$. Then the short distance behavior $t \rightarrow 0$ of $\tau(t)$ is given by 


$$
\begin{aligned}
e^{t} \tau(t) \sim \text { const. } t^{\left(\sigma^{2}-\theta^{2}\right) / 4}(1+ & \frac{1}{2 \sigma^{2}}\left(\sigma^{2}-\theta^{2}\right) t-\frac{\sigma^{2}-\theta^{2}}{4 \sigma^{2}(1+\sigma)^{2}} \hat{s} t^{1+\sigma} \\
& \left.-\frac{\sigma^{2}-\theta^{2}}{4 \sigma^{2}(1-\sigma)^{2}} \hat{s}^{-1} t^{1-\sigma}+\cdots\right) \quad(\text { if } \quad \sigma \neq 0) \\
& \sim \text { const. } t^{-\theta^{2} / 4}\left(1+\frac{\theta^{2}}{4} t\left(\Omega^{2}+2 \Omega+3\right)+\cdots\right) \quad(\text { if } \quad \sigma=0)
\end{aligned}
$$

where

$$
\begin{gathered}
s=\frac{\Gamma(1-\sigma)^{2} \Gamma\left(1+\frac{\sigma+\theta}{2}\right) \Gamma\left(1+\frac{\sigma-\theta}{2}\right)}{\Gamma(1+\sigma)^{2} \Gamma\left(1-\frac{\sigma+\theta}{2}\right) \Gamma\left(1-\frac{\sigma-\theta}{2}\right)}, \\
\Omega=1-\psi\left(1+\frac{\theta}{2}\right)-\psi\left(1-\frac{\theta}{2}\right)+4 \psi(1)-\log t .
\end{gathered}
$$

The particular case $\theta=0$ agrees with the result of McCoy-Tracy-Wu [3] (for $v=0$ ).

\section{Referemces}

[1] Myers, J. M., J. Math. Phys. 6 (1965), 1839.

[2] Wu, T. T., McCoy, B. M. Tracy C. A. and Barouch E., Phys. Rev. $B 13$ (1976), 316.

[3] McCoy, B. M., Tracy C. A. and Wu T. T., J. Math. Phys. 18 (1977), 1058.

[4] Ablowitz, M. and Segur H., Phys. Rev. Lett. 38 (1977), 1103. Ablowitz M., Ramani A. and Segur H., J. Math. Phys. 21 (1980), 715, 1006.

[ 5] Sato, M., Miwa T. and Jimbo M., Publ. RIMS. Kyoto Univ. 14 (1978), 223, 15 (1979), 201, 577, 871, 16 (1980), 531, 17 (1981), 137.

[6] Jimbo, M., Miwa T., Môri Y. and Sato M., Physica $1 D$ (1980), 80.

[ 7 ] Jimbo, M., Miwa T. and Ueno K., Physica 2D (1981), 245. Jimbo M. and Miwa T., Physica 2D (1981), 407, ibid. 4D (1981), 26.

[8] Miwa, T., Publ. RIMS, Kyoto Univ. 17 (1981), 665, 703.

[9] Garnier, R., Ann. Ecol. Norm. Sup. 29 (1912), 1.

[10] Okamoto, K., Physica 2D (1981), 525.

[11] Erdélyi, A., Higher transcendental functions, 1, McGraw-Hill, 1953. 
